\title{
A local discontinuous Galerkin method for the (non)-isothermal Navier-Stokes-Korteweg equations
}

\author{
Lulu Tian ${ }^{\mathrm{a}}$, Yan $\mathrm{Xu}^{\mathrm{b}, *}$, J.G.M. Kuerten ${ }^{\mathrm{a}, \mathrm{c}}$, J.J.W. van der Vegt ${ }^{\mathrm{a}}$ \\ ${ }^{a}$ Mathematics of Computational Science Group \\ Dept. of Applied Mathematics, University of Twente, \\ P.O. Box 217, 7500 AE, Enschede, The Netherlands. \\ ${ }^{b}$ School of Mathematical Sciences, University of Science and Technology of China, \\ Hefei, Anhui 230026, P.R. China. \\ ${ }^{c}$ Computational Multiphase Flow \\ Dept. of Mechanical Engineering, Eindhoven University of Technology, \\ P.O. Box 513, 5600 MB Eindhoven, The Netherlands.
}

\begin{abstract}
In this article, we develop a local discontinuous Galerkin (LDG) discretization of the (non)-isothermal Navier-Stokes-Korteweg (NSK) equations in conservative form. These equations are used to model the dynamics of a compressible fluid exhibiting liquid-vapour phase transitions. T he NSK-equations are closed with a Van der Waals equation of state and contain third order nonlinear derivative terms. These contributions frequently cause standard numerical methods to violate the energy dissipation relation and require additional stabilization terms to prevent numerical instabilities. In order to address these problems we first develop an LDG method for the isothermal NSK equations using discontinuous finite element spaces combined with a time-implicit Runge-Kutta integration method. Next, we extend the LDG discretization to the non-isothermal NSK equations. An important feature of the LDG discretizations presented in this article is that they are relatively simple, robust and do not require special regularization terms. Finally, computational experiments are provided to demonstrate the capabilities, accuracy and stability of the LDG discretizations.
\end{abstract}

\footnotetext{
${ }^{*}$ Corresponding author

Email addresses: 1.tian@utwente.nl (Lulu Tian), yxu@ustc.edu.cn (Yan Xu), J.G.M.Kuerten@tue.nl ( J.G.M. Kuerten), j.j.w.vandervegt@utwente.nl (J.J.W. van der Vegt)
} 
Keywords: local discontinuous Galerkin method, (non-)isothermal Navier-Stokes-Korteweg equations, phase transition, Van der Waals equation of state, implicit time integration, accuracy and stability

\section{Introduction}

In this article, we will present a local discontinuous Galerkin (LDG) method for the numerical solution of the Navier-Stokes-Korteweg (NSK) equations that model liquid-vapor phase transitions. This research is motivated by our previous work [35], where we solved a mixed hyperbolicelliptic system that models phase transitions in solids and fluids using an LDG method. In that article, $L_{2}-$ stability of the LDG discretization of the phase transition model was proved, and an error estimate for the LDG discretization for the viscosity-capillarity (VC) system with linear strain-stress relation was provided. The numerical experiments discussed in [35] show that the LDG method for the $\mathrm{VC}$ system is stable and the LDG solutions converge to the analytical solution of the original problem.

Two-phase flows can be treated either by sharp interface models or by diffuse interface models. Sharp interface models assume that the interface thickness is equal to zero and have successfully been applied to many twophase flows $[4,11,10,36]$. Sharp interface models require, however, an extra evolution equation for the interface and face challenges in the reconstruction of the interface, leading to mathematical models that are solved by a Level Set or a Volume of Fluid method [23]. In contrast, diffuse interface models [3, $6,40,33]$ regard the interface as thin layers of fluid where properties such as mass density, viscosity and pressure change smoothly. In the diffuse interface model, only a single set of governing equations needs to be solved on the entire flow domain, including the interface area. The Navier-Stokes-Korteweg (NSK) equations [17, 31, 32, 5, 29] contain an additional contribution to the stress tensor related to capillary forces and are an example of a diffuse interface model. The NSK equations are used in this article to model the dynamics of a compressible fluid exhibiting phase transitions between liquid and vapour.

We consider a fluid in a domain $\Omega \in \mathbb{R}^{d}$ with $d \leq 3$, and let $\rho$ be the density of the fluid and $\mathbf{u}$ the velocity. The isothermal NSK equations with 
zero external forces, in dimensionless and conservative form, read

$$
\begin{array}{r}
\frac{\partial \rho}{\partial t}+\nabla \cdot(\rho \mathbf{u})=0 \\
\frac{\partial(\rho \mathbf{u})}{\partial t}+\nabla \cdot(\rho \mathbf{u} \otimes \mathbf{u}+p \mathbf{I})-\nabla \cdot \tau-\nabla \cdot \xi=\mathbf{0}
\end{array}
$$

in $\Omega \times(0, T]$, with $p$ the pressure, $\otimes$ the tensor product and $\mathbf{I}$ the identity matrix. The viscous stress tensor $\tau$ and Korteweg stress tensor $\xi$ are given by

$$
\begin{array}{r}
\tau=\frac{1}{\mathbb{R} e}\left(\nabla \mathbf{u}+\nabla^{T} \mathbf{u}-\frac{2}{3} \nabla \cdot \mathbf{u I}\right), \\
\xi=\frac{1}{\mathbb{W} e}\left(\left(\rho \triangle \rho+\frac{1}{2}|\nabla \rho|^{2}\right) \mathbf{I}-\nabla \rho \nabla^{T} \rho\right),
\end{array}
$$

where $\mathbb{R} e, \mathbb{W} e$ are the Reynolds number and Weber number. The definition of the dimensionless variables is summarized in the Appendix. To simulate phase transitions between liquid and vapour, which are distinguished by different values of the density $\rho$, we need an expression for the thermodynamic pressure that is valid in both liquid and vapour state. The Van der Waals equation of state is an appropriate choice, especially close to the critical temperature. For the isothermal NSK equations, we use the following dimensionless form $[29,16]$

$$
p(\theta, \rho)=\frac{8}{27} \frac{\theta \rho}{(1-\rho)}-\rho^{2},
$$

with $\theta$ the dimensionless temperature. Figure 1 describes the shape of the Van der Waals type equation of state (3) for temperature $\theta=0.85$.

Other relevant thermodynamic quantities for (non)-isothermal fluids [29, 14] are the free energy density

$$
W(\rho, \theta)=R \theta \rho \log \left(\frac{\rho}{b-\rho}\right)-a \rho^{2},
$$

and the chemical potential

$$
\mu(\rho, \theta)=R \theta \log \left(\frac{\rho}{b-\rho}\right)+R \theta \frac{b}{b-\rho}-2 a \rho .
$$


For isothermal flows, the total energy can be defined as

$$
\mathcal{E}(\rho, \rho \mathbf{u})=\int_{\Omega}\left(W(\rho)+\frac{1}{2 \mathbb{W} e}|\nabla \rho|^{2}+\frac{1}{2} \frac{|\rho \mathbf{u}|^{2}}{\rho}\right) d \mathbf{x}
$$

and satisfies for periodic boundary conditions the relation $[29,16,5,33]$

$$
\frac{d}{d t} \mathcal{E}(\rho(\cdot, t), \rho \mathbf{u}(\cdot, t))=-\int_{\Omega} \nabla \mathbf{u}: \tau d \mathbf{x} \leq 0,
$$

for positive Re. Here ":" is summation of the element-wise product of two matrices. Suppose $A=\left(a_{i j}\right), B=\left(b_{i j}\right) \in \mathbb{R}^{d \times d}$, then $A: B=\sum_{i} \sum_{j} a_{i j} b_{i j}$.

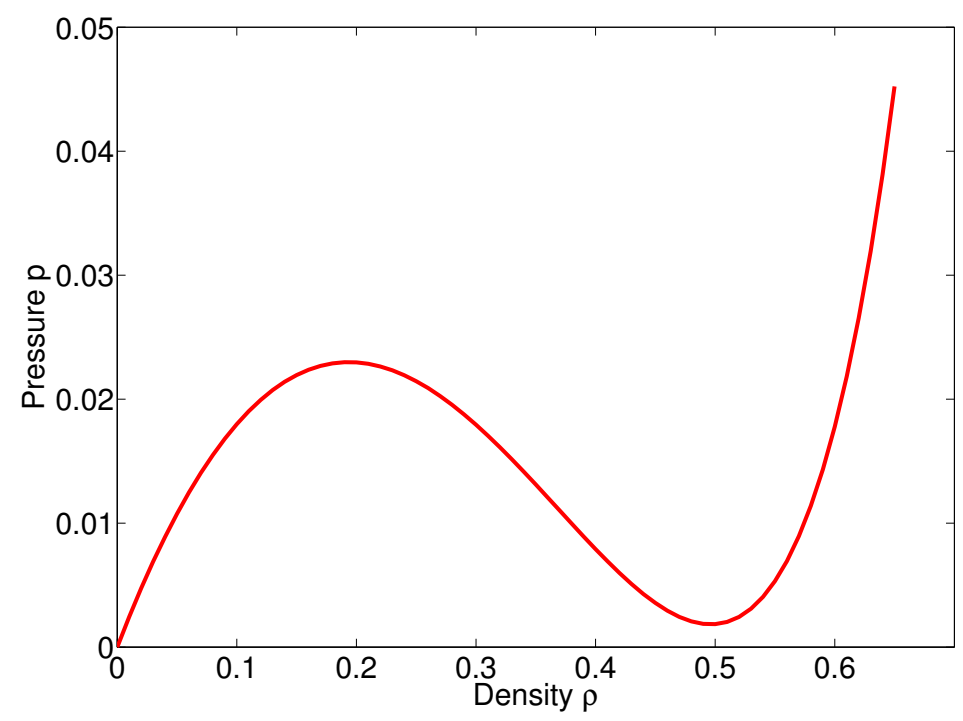

Figure 1: Van der Waals type of pressure-density relation at the dimensionless temperature $\theta=0.85$, gas constant $R=\frac{8}{27}$, and coefficients $a=1.0, b=1.0$.

An important question is the solvability of the isothermal NSK equations, which has received considerable attention. For isothermal NSK equations, local and global smooth solutions for Cauchy problems of (1) with constant coefficients and small, smooth initial data were discussed in [21, 22]; the extension to Lipschitz continuous viscous coefficients and more general initial conditions was presented in [26]. In [33] a mathematical model with physically relevant non-local energies was proposed instead of the Van der Waals 
free energy and a short-time existence theorem for the Cauchy problem of the non-local NSK equations was proved. The existence of strong solutions and global weak solutions of the isothermal NSK system (1) modeling compressible fluids of Korteweg type was discussed in [12, 20].

As discussed in [1,35], it is not trivial to obtain a numerical solution of mixed hyperbolic-elliptic systems. When it comes to the more complex mixed system (1), the non-monotonic Van der Waals equation of state can induce instabilities in the numerical solution. And the third order spatial derivatives of the mass density, stemming from the divergence of the Korteweg tensor $\xi$, causes dispersive behaviour of the numerical solution. Therefore numerical methods for the isothermal NSK equations face several challenges. One difficulty is that standard numerical methods including finite difference, finite volume, and discontinuous Galerkin (DG) methods with poor numerical fluxes, may violate the energy dissipation relation (5) and suffer from an increase in energy for multiphase flows [14]. Another problem is the occurrence of parasitic currents: unphysical velocities close to the interface. In particular, the velocity field does not tend to zero when equilibrium is approached $[14,16]$. In [24], a method is presented to eliminate parasitic currents for finite volume methods, but this is still a topic of ongoing research. Moreover, to capture the interface accurately requires locally a fine mesh.

Many articles addressed the numerical solution of the isothermal NavierStokes-Korteweg equations modeling liquid-vapour flows with phase change. A detailed description of higher order schemes, including the local discontinuous Galerkin method, to solve the non-conservative form of the isothermal NSK equations was given in [14]. A finite element formulation based on an isogeometric analysis of the non-conservative form of the isothermal NSK equations was developed in [17]. This method can straightforwardly deal with the higher-order derivatives in the isothermal NSK equations. In [29] a semi-discrete Galerkin method based on entropy variables and a new time integration scheme was proposed for the non-conservative form of the isothermal NSK equations. A DG scheme for the non-conservative form of the isothermal NSK equations, obtained by choosing special numerical fluxes, was presented in [16]. Another way to obtain a stable numerical discretization of the isothermal NSK equations is by adding two vanishing regularization terms. This approach was successfully used in [5] in combination with globally continuous finite element spaces and a time-implicit discretization.

The non-isothermal NSK equations [3, 32] model two-phase flows involving phase transition at nonuniform temperatures. Besides the isothermal 
equations (1), the non-isothermal NSK equations also contain an equation for the total energy

$$
\begin{array}{r}
\frac{\partial \rho}{\partial t}+\nabla \cdot(\rho \mathbf{u})=0, \\
\frac{\partial \rho \mathbf{u}}{\partial t}+\nabla \cdot(\rho \mathbf{u} \otimes \mathbf{u}+p \mathbf{I})-\nabla \cdot \tau-\nabla \cdot \xi=\mathbf{0}, \\
\frac{\partial(\rho E)}{\partial t}+\nabla \cdot((\rho E+p) \mathbf{u})-\nabla \cdot((\tau+\xi) \cdot \mathbf{u})+\nabla \cdot \mathbf{q}+\nabla \cdot \mathbf{j}_{E}=0,
\end{array}
$$

where the total energy density is given by

$$
\rho E=\rho e+\frac{1}{2} \rho|\mathbf{u}|^{2}+\frac{1}{2} \frac{1}{\mathbb{W} e}|\nabla \rho|^{2} .
$$

For the non-isothermal NSK equations (6), the dimensionless Van der Waals equation of state is given by $[32,13]$

$$
p=\frac{8 \theta \rho}{3-\rho}-3 \rho^{2}
$$

The specific internal energy $e$ in (7) is given by

$$
e=\frac{8}{3} C_{v} \theta-3 \rho
$$

with $C_{v}$ the specific heat at constant volume. The total entropy is specified as $S=\rho s$ with $s$ the entropy density

$$
s=-\frac{8}{3} \log \left(\frac{\rho}{3-\rho}\right)+\frac{8}{3} C_{v} \log (\theta) .
$$

The heat flux $\mathbf{q}$ and energy flux $\mathbf{j}_{E}$ through the interface in (6) are defined as

$$
\mathbf{q}=-\frac{8 C_{v}}{3 \mathbb{W} e \mathbb{P} r} \nabla \theta, \quad \mathbf{j}_{E}=\frac{1}{\mathbb{W} e}(\rho \nabla \cdot \mathbf{u}) \nabla \rho
$$

where $\mathbb{P r}$ is the Prandtl number, see Appendix. Recently global existence and uniqueness of strong solutions to the non-isothermal NSK equations were proved for bounded domains in [27, 28].

Most articles so far only discuss the non-conservative form of the isothermal NSK equations (1). Frequently, the isothermal NSK equations (1) are 
rewritten into an extended system by adding an extra variable for the total energy equation $[29,16,5,33]$. It is, however, not trivial to do this for the non-isothermal NSK equations, where the Van der Waals equation of state depends on both the density and temperature, so the frequently used relation

$$
\nabla p=\rho \nabla W^{\prime}(\rho)
$$

is no longer satisfied. The numerical methods for the isothermal NSK equations discussed in $[29,16,5,33]$ are therefore difficult to extend to the nonisothermal NSK equations. The great potential of the LDG method to solve phase transition problems, as shown in $[18,35]$, motivated us to develop an LDG method for systems (1) and (6), while keeping the conservative form of the (non)-isothermal NSK equations and obtain a stable numerical discretization without additional regularization terms. T o our knowledge, we are the first to discuss an LDG method for the conservative form of the (non)-isothermal NSK equations.

The LDG method is an extension of the discontinuous Galerkin (DG) method that aims to solve partial differential equations (PDEs) containing higher order spatial derivatives and was originally developed by Cockburn and Shu in [9] for solving nonlinear convection-diffusion equations containing second-order spatial derivatives. The idea behind LDG methods is to rewrite the original equations as a first order system, and then apply the DG method to this first order system. The design of the numerical fluxes is the key ingredient to ensure stability. LDG techniques have been developed for convection-diffusion equations [9], nonlinear KdV type equations [39], the Camassa-Holm equation [37] and many other types of partial differential equations. For a review, see [38]. The LDG method results in an extremely local discretization, which offers great advantages in parallel computing and $h p$-adaptation.

The organization of this article is as follows. In Section 2 we present the LDG method for the (non)-isothermal NSK equations in detail. An important aspect of this discretization is that it preserves the conservative form of the NSK equations. Section 3 discusses an implicit Runge-Kutta time integration method, which is used to overcome the stiffness of the NSK equations. In Section 4, numerical experiments are presented to investigate the accuracy and stability of the LDG discretization of the (non)-isothermal NSK equations. For the isothermal NSK equations, discrete mass conservation and energy dissipation are verified for the LDG solutions, while the 
mass conservation and total entropy are verified for the non-isothermal NSK equations. Finally, we give conclusions in Section 5 .

\section{LDG Discretization of the NSK system}

In this section, we develop an LDG method to solve the (non)-isothermal NSK system in $\Omega \in \mathbb{R}^{d}$ with $d \leq 3$. We restrict the numerical experiments to one and two dimensions in this paper, but the LDG discretization described here can be easily extended to three dimensions. We first introduce notations used for the description of the LDG discretization.

\subsection{Notations}

We denote by $\mathcal{T}_{h}$ a tessellation of $\Omega$ with regular shaped elements $K, \Gamma$ represents all boundary faces of $K \in \mathcal{T}_{h}$ and $\Gamma_{0}=\Gamma \backslash \partial \Omega$. Suppose $e$ is a face shared by the "left" and "right" elements $K_{L}$ and $K_{R}$. The normal vectors $\mathbf{n}_{L}$ and $\mathbf{n}_{R}$ on $e$ point, respectively, exterior to $K_{L}$ and $K_{R}$. Let $\varphi$ be a function on $K_{L}$ and $K_{R}$, which could be discontinuous across $e$, then the left and right trace are denoted as $\varphi_{L}=\left.\left(\left.\varphi\right|_{K_{L}}\right)\right|_{e}, \varphi_{R}=\left.\left(\left.\varphi\right|_{K_{R}}\right)\right|_{e}$, respectively. For more details about these definitions, we refer the reader to [38].

For the LDG discretization, we define the finite element spaces

$$
\begin{aligned}
V_{h}= & \left\{\phi \in L^{2}(\Omega):\left.\phi\right|_{K} \in \mathcal{P}^{k}(K), \forall K \in \mathcal{T}_{h}\right\}, \\
\Sigma_{h}^{d}= & \left\{\boldsymbol{\Phi}=\left(\phi^{(1)}, \phi^{(2)}, \ldots, \phi^{(d)}\right)^{T} \in\left(L^{2}(\Omega)\right)^{d}:\left.\phi^{(i)}\right|_{K} \in \mathcal{P}^{k}(K),\right. \\
& \left.i=1, \ldots, d, \forall K \in \mathcal{T}_{h}\right\},
\end{aligned}
$$

with $\mathcal{P}^{k}(K)$ the space of polynomials of degree up to $k \geq 0$ on $K \in \mathcal{T}_{h}$.

The numerical solution is denoted by $\mathbf{U}_{h}$, with each component of $\mathbf{U}_{h}$ belonging to the finite element space $V_{h}$, and can be written as

$$
\left.\mathbf{U}_{h}(\mathbf{x}, t)\right|_{K}=\sum_{l=0}^{N_{p}} \widehat{\mathbf{U}}_{l}^{K}(t) \phi_{l}(\mathbf{x}), \text { for } \mathbf{x} \in K \text {. }
$$

Here, $\widehat{\mathbf{U}}_{l}^{K}(t)$ are unknowns and Legendre polynomials are used for the basis functions $\phi_{l}(\mathbf{x})$. In the next two sections we will discuss a local discontinuous Galerkin discretization for both the isothermal and non-isothermal NSK equations. We first consider the isothermal NSK equations since they provide a simpler model for phase transitions and are frequently used in applications. The non-isothermal NSK equations are computationally more demanding, but provide a more realistic model of the complex physics of phase transition. 


\section{2. $L D G$ discretization for the isothermal NSK equations}

In this section, we propose an LDG discretization for the isothermal NSK equations, which are rewritten as a first order system, given by the primary equations

$$
\begin{aligned}
& \rho_{t}+\nabla \cdot \mathbf{m}=0, \\
& \mathbf{m}_{t}+\nabla \cdot \mathbf{F}(\mathbf{U})-\nabla \cdot \tau(\mathbf{z}, s)-\nabla \cdot \xi(\rho, \mathbf{r}, g)=\mathbf{0},
\end{aligned}
$$

and auxiliary equations

$$
\begin{aligned}
& \mathbf{z}=\nabla \mathbf{u}, \\
& l=\nabla \cdot \mathbf{u}, \\
& \mathbf{r}=\nabla \rho, \\
& g=\nabla \cdot \mathbf{r},
\end{aligned}
$$

where

$$
\begin{aligned}
\mathbf{u} & =\frac{\mathbf{m}}{\rho}, \\
\tau & =\frac{1}{\mathbb{R} e}\left(\mathbf{z}+\mathbf{z}^{T}-\frac{2}{3} l \mathbf{I}\right), \\
\xi & =\frac{1}{\mathbb{W} e}\left(\left(\rho g+\frac{1}{2}|\mathbf{r}|^{2}\right) \mathbf{I}-\mathbf{r r}^{T}\right),
\end{aligned}
$$

and

$$
\mathbf{F}(\mathbf{U})=\mathbf{m} \otimes \mathbf{u}+p(\rho) \mathbf{I}, \quad \mathbf{U}=\left(\begin{array}{c}
\rho \\
\mathbf{m}
\end{array}\right)
$$

The LDG discretization for the isothermal NSK equations (11) - (13) is now as follows: find $\rho_{h}, l_{h}, g_{h} \in V_{h}$, and $\mathbf{m}_{h}, \mathbf{z}_{h}, \mathbf{r}_{h} \in \Sigma_{h}^{d}$, such that for all test functions $\phi, \varphi, \zeta \in V_{h}$ and $\psi, \eta, \varsigma \in \Sigma_{h}^{d}$, the following relations are satisfied

$$
\begin{aligned}
& \int_{K}\left(\rho_{h}\right)_{t} \phi d K-\int_{K} \mathbf{m}_{h} \cdot \nabla \phi d K+\int_{\partial K} \widehat{\mathbf{m}_{h}} \cdot \mathbf{n} \phi d s=0, \\
& \int_{K}\left(\mathbf{m}_{h}\right)_{t} \psi d K-\int_{K}\left(\mathbf{F}_{h}-\tau_{h}-\xi_{h}\right) \cdot \nabla \psi d K+\int_{\partial K}\left(\widehat{\mathbf{F}_{h}}-\widehat{\tau_{h}}-\widehat{\xi_{h}}\right) \cdot \mathbf{n} \psi d s=\mathbf{0},
\end{aligned}
$$


and

$$
\begin{aligned}
& \int_{K} \mathbf{z}_{h} \eta d K=-\int_{K} \mathbf{u}_{h} \nabla \cdot \eta d K+\int_{\partial K} \widehat{\mathbf{u}_{h}} \eta \cdot \mathbf{n} d s, \\
& \int_{K} l_{h} \zeta d K=-\int_{K} \mathbf{u}_{h} \cdot \nabla \zeta d K+\int_{\partial K} \widehat{\mathbf{u}_{h}} \cdot \mathbf{n} \zeta d s \\
& \int_{\mathbf{r}_{h} \varsigma d K}=-\int_{K} \rho_{h} \nabla \cdot \varsigma d K+\int_{\partial K} \widehat{\rho_{h}} \varsigma \cdot \mathbf{n} d s, \\
& \int_{K} g_{h} \varphi d K=-\int_{K} \mathbf{r}_{h} \cdot \nabla \varphi d K+\int_{\partial K} \widehat{\mathbf{r}_{h}} \cdot \mathbf{n} \varphi d s,
\end{aligned}
$$

where

$$
\begin{aligned}
\widehat{\mathbf{u}_{h}} & =\frac{\widehat{\mathbf{m}_{h}}}{\widehat{\rho}_{h}} \\
\widehat{\tau_{h}} & =\frac{1}{\mathbb{R e}}\left(\widehat{\mathbf{z}_{h}}+\widehat{\mathbf{z}}^{T}-\frac{2}{3} \widehat{l}_{h} \mathbf{I}\right), \\
\widehat{\xi_{h}} & =\frac{1}{\mathbb{W} e}\left(\left(\widehat{\rho_{h}} \widehat{g_{h}}+\frac{1}{2}\left|\widehat{\mathbf{r}}_{h}\right|^{2}\right) I-\widehat{\mathbf{r}}_{h} \widehat{\mathbf{r}}_{h}^{T}\right),
\end{aligned}
$$

and

$$
\mathbf{F}_{h}=\mathbf{F}\left(\mathbf{U}_{h}\right), \quad \mathbf{U}_{h}=\left(\begin{array}{c}
\rho_{h} \\
\mathbf{m}_{h}
\end{array}\right) .
$$

For the numerical fluxes in (14), (15) and (16), denoted with a hat, we choose the Lax-Friedrich flux for the convective part and central numerical fluxes for the other terms,

$$
\begin{aligned}
& \left.\widehat{\mathcal{F}_{h}}\right|_{e}=\frac{1}{2}\left(\left.\mathcal{F}_{h}\right|_{L}+\left.\mathcal{F}_{h}\right|_{R}-\alpha\left(\left.\mathbf{U}_{h}\right|_{R}-\left.\mathbf{U}_{h}\right|_{L}\right)\right), \quad \text { with } \mathcal{F}_{h}=\left(\begin{array}{c}
\mathbf{m}_{h} \\
\mathbf{F}_{h}
\end{array}\right), \\
& \left.\widehat{\mathbf{m}_{h}}\right|_{e}=\frac{1}{2}\left(\left.\mathbf{m}_{h}\right|_{L}+\left.\mathbf{m}_{h}\right|_{R}\right),\left.\quad \widehat{\rho}_{h}\right|_{e}=\frac{1}{2}\left(\left.\rho_{h}\right|_{L}+\left.\rho_{h}\right|_{R}\right), \\
& \left.\widehat{\mathbf{r}_{h}}\right|_{e}=\frac{1}{2}\left(\left.\mathbf{r}_{h}\right|_{L}+\left.\mathbf{r}_{h}\right|_{R}\right),\left.\quad \widehat{\mathbf{z}_{h}}\right|_{e}=\frac{1}{2}\left(\left.\mathbf{z}_{h}\right|_{L}+\left.\mathbf{z}_{h}\right|_{R}\right), \\
& \left.\widehat{l}_{h}\right|_{e}=\frac{1}{2}\left(\left.l_{h}\right|_{L}+\left.l_{h}\right|_{R}\right),\left.\quad \widehat{g}_{h}\right|_{e}=\frac{1}{2}\left(\left.g_{h}\right|_{L}+\left.g_{h}\right|_{R}\right),
\end{aligned}
$$

with $\alpha$ a positive constant that is chosen as the maximum absolute eigenvalue of $\frac{\partial \mathcal{F}_{h}}{\partial \mathbf{U}_{h}}$ globally. 


\section{3. $L D G$ Discretization for the non-isothermal NSK equations}

The LDG discretization for the isothermal NSK equations presented in Section 2.2 can be extended to the non-isothermal NSK equations (6). The equations can also be rewritten as a first order system that includes Equations (11)-(13) and additional equations given by

$$
\begin{aligned}
& (\rho E)_{t}+\nabla \cdot \mathbf{G}(\mathbf{U})-\nabla \cdot((\tau+\xi) \cdot \mathbf{u})+\nabla \cdot \mathbf{q}+\nabla \cdot \mathbf{j}_{E}=0 \\
& \mathbf{q}=-\frac{8 C_{v}}{3 \mathbb{W} e \mathbb{P} r} \nabla \theta \\
& \mathbf{j}_{E}=\frac{1}{\mathbb{W} e} \rho l \mathbf{r}, \quad \mathbf{G}(\mathbf{U})=(\rho E+p) \mathbf{u} .
\end{aligned}
$$

The LDG discretization for the non-isothermal NSK equations contains (14)-(16), and the LDG discretization for the energy equation contributions, given by (18), can be written as

$$
\begin{gathered}
\int_{K}\left((\rho E)_{h}\right)_{t} \chi d K-\int_{K}\left(\mathbf{G}_{h}-\left(\tau_{h}+\xi_{h}\right) \cdot \mathbf{u}_{h}+\mathbf{q}_{h}+\left(\mathbf{j}_{E}\right)_{h}\right) \cdot \nabla \chi d K+ \\
\left.\int_{\partial K}\left(\widehat{\mathbf{G}_{h}}-\left(\widehat{\tau_{h}}+\widehat{\xi_{h}}\right) \cdot \widehat{\mathbf{u}_{h}}+\widehat{\mathbf{q}_{h}}+\widehat{\left(\mathbf{j}_{E}\right.}\right)_{h}\right) \cdot \mathbf{n} \chi d s=0 . \\
\int_{K} \mathbf{q}_{h} \sigma d K-\frac{8 C_{v}}{3 \mathbb{W} e \mathbb{P r}} \int_{K} \theta_{h} \nabla \cdot \sigma d K+\frac{8 C_{v}}{3 \mathbb{W} e \mathbb{P r}} \int_{\partial K} \widehat{\theta_{h}} \sigma \cdot \mathbf{n} d s=0,
\end{gathered}
$$

with

$$
\left(\mathbf{j}_{E}\right)_{h}=\frac{1}{\mathbb{W} e} \rho_{h} l_{h} \mathbf{r}_{h}, \mathbf{G}_{h}=\mathbf{G}\left(\mathbf{U}_{h}\right) \text { and } \mathbf{U}_{h}=\left(\begin{array}{c}
\rho_{h} \\
\mathbf{m}_{h} \\
(\rho E)_{h}
\end{array}\right)
$$

The same numerical fluxes (17) as used in the LDG discretization for the isothermal NSK equations are used in the non-isothermal equations. If we denote $\mathcal{G}_{h}\left(\mathbf{U}_{h}\right)=\left(\begin{array}{c}\mathcal{F}_{h} \\ \mathbf{G}_{h}\end{array}\right)$, then the numerical flux for $\mathcal{G}_{h}$ is also the LaxFriedrichs flux, but now with the constant $\alpha$ the maximum absolute eigenvalue of $\frac{\partial \mathcal{G}_{h}}{\partial \mathbf{U}_{h}}$. For the one dimensional case the eigenvalues of $\frac{\partial \mathcal{G}_{h}}{\partial \mathbf{U}_{h}}$ are provided in [13]. For two dimensional case, $u, v$ are additional eigenvalues in, respectively $x$ and $y$ direction. The additional numerical fluxes in the energy equation contributions (20) are chosen as

$$
\widehat{\mathbf{q}_{h}}=\frac{1}{2}\left(\left.\mathbf{q}_{h}\right|_{L}+\left.\mathbf{q}_{h}\right|_{R}\right), \quad \widehat{\theta_{h}}=\frac{1}{2}\left(\left.\theta_{h}\right|_{L}+\left.\theta_{h}\right|_{R}\right), \quad \widehat{\left(\mathbf{j}_{E}\right)_{h}}=\frac{1}{W e} \widehat{\rho_{h}} \widehat{l_{h}} \widehat{\mathbf{r}_{h}}
$$


Remark 2.1. We emphasize that not only the (first-order) convective part of the isothermal NSK equations (11), but also the (first-order) convective part of the non-isothermal NSK equations is of mixed hyperbolic-elliptic type. The eigenvalues of the systems are given explicitly below.

The convective part of the isothermal NSK equations is given by

$$
\begin{aligned}
\frac{\partial \rho}{\partial t}+\nabla \cdot(\rho \mathbf{u}) & =0 \\
\frac{\partial(\rho \mathbf{u})}{\partial t}+\nabla \cdot(\rho \mathbf{u} \otimes \mathbf{u}+p) & =0 .
\end{aligned}
$$

In $2 D$, assuming $\mathbf{u}=(u, v)^{T}$, the Jacobian matrices are

$$
A^{1}=\left(\begin{array}{ccc}
0 & 1 & 0 \\
-u^{2}+p^{\prime}(\rho) & 2 u & 0 \\
-u v & v & u
\end{array}\right), \quad A^{2}=\left(\begin{array}{ccc}
0 & 0 & 1 \\
-u v & v & u \\
-v^{2}+p^{\prime}(\rho) & 0 & 2 v
\end{array}\right)
$$

for, respectively, the $x$ - and $y$-direction. The eigenvalues of $A^{1}$ are $\{u-$ $\left.\sqrt{p^{\prime}(\rho)}, u, u+\sqrt{p^{\prime}(\rho)}\right\}$ and those of $A^{2}$ are $\left\{v-\sqrt{p^{\prime}(\rho)}, v, v+\sqrt{p^{\prime}(\rho)}\right\}$. Consequently, (22) is a hyperbolic-elliptic system due to the non-monotonicity of the Van der Waals equation of state, and it is elliptic when $p^{\prime}(\rho)<0$.

The eigenvalues of the convective part of the one dimensional non-isothermal NSK equations were studied in [13]. The eigenvalues of the 2D non-isothermal NSK equations are the extension of those of the $1 D$ non-isothermal NSK equations, with one extra $u, v$, in the $x, y$ direction respectively. In detail, the convective part of the non-isothermal NSK equations is rewritten, in primitive form, as

$$
\mathbf{V}_{t}+\widetilde{F}(\mathbf{V})_{x}+\widetilde{G}(\mathbf{V})_{y}=0, \text { with } \mathbf{V}=(\rho, u, v, p) \text { in } 2 D
$$

The Jacobian matrices are given by

$$
A^{1}=\frac{\partial \widetilde{F}}{\partial \mathbf{V}}=\left(\begin{array}{cccc}
u & \rho & 0 & 0 \\
0 & u & 0 & 1 / \rho \\
0 & 0 & u & 0 \\
0 & f(p, \rho) & 0 & u
\end{array}\right), \quad A^{2}=\frac{\partial \widetilde{G}}{\partial \mathbf{V}}=\left(\begin{array}{cccc}
v & 0 & \rho & 0 \\
0 & v & 0 & 0 \\
0 & 0 & v & 1 / \rho \\
0 & 0 & f(p, \rho) & v
\end{array}\right),
$$


where the function $f$ is equal to

$$
f(p, \rho)=\frac{3}{C_{v}(3-\rho)}\left(\left(1+C_{v} p+\rho^{2}\left(3-3 C_{v}+2 C_{v} \rho\right)\right)\right) .
$$

The eigenvalues of $A^{1}$ are $\{u, u, u-\sqrt{\beta}, u+\sqrt{\beta}\}$, and those of $A^{2}$ are $\{v, v, v-\sqrt{\beta}, v+\sqrt{\beta}\}$, with $\beta=2\left(\frac{p}{\rho}+\frac{4 \theta\left(3+C_{v}(-3+2 \rho)\right)}{C_{v}(-3+\rho)^{2}}\right)$.

\section{Implicit time discretization method}

The equations for the DG expansion coefficients for each variable are obtained by introducing the polynomial representation (10) into the LDG discretizations (14)-(16) and (20). The coefficients of the polynomial expansions of $\rho_{h}(\mathbf{x}, t), \mathbf{m}_{h}(\mathbf{x}, t)$ and $\rho E_{h}(\mathbf{x}, t)$ in the LDG discretization are collected in the vector $\widehat{\mathbf{U}}(t)$. The LDG discretization, given by (14)-(16) and (20) with corresponding fluxes for $\rho_{h}(\mathbf{x}, t), \mathbf{m}_{h}(\mathbf{x}, t)$ and $\rho E_{h}(\mathbf{x}, t)$, then results in a system of ordinary differential and algebraic equations (DAEs)

$$
\begin{aligned}
& \frac{d \widehat{\mathbf{U}}(t)}{d t}+\mathbf{L}(\widehat{\mathbf{U}}(t), \widehat{\mathbf{Z}}(t), \widehat{\mathbf{G}}(t))=0, \\
& \widehat{\mathbf{Z}}(t)-\mathbf{P}(\widehat{\mathbf{U}}(t))=0, \\
& \widehat{\mathbf{G}}(t)-\mathbf{Q}(\widehat{\mathbf{U}}(t), \widehat{\mathbf{Z}}(t))=0,
\end{aligned}
$$

with $\widehat{\mathbf{Z}}(t), \widehat{\mathbf{G}}(t)$ the coefficients of the auxiliary variables, and $\mathbf{L}, \mathbf{P}, \mathbf{Q}$ nonlinear functions of $\widehat{\mathbf{U}}(t), \widehat{\mathbf{Z}}(t)$, and $\widehat{\mathbf{G}}(t)$. The initial values are

$$
\widehat{\mathbf{U}}\left(t_{0}\right)=\widehat{\mathbf{U}}_{0} \text {. }
$$

Note, in case of the isothermal NSK equations, the contributions from (20) are missing. Both explicit and implicit time integration methods [19] can be applied to the DAE system (23). Due to the Korteweg stress tensor $\xi$, the NSK system is a third order nonlinear system of partial differential equations, and explicit time integration methods then require the time step to satisfy

$$
\Delta t=O\left(h^{2}\right), \quad h=\min \{\Delta x, \Delta y\},
$$

for stability [13]. Moreover, when we would consider local mesh refinement near the interface, the time step restriction (24) becomes even more severe. Therefore, we consider an implicit time stepping method. 


\subsection{Diagonally Implicit Runge-Kutta methods}

For the implicit time integration we use Diagonally Implicit Runge-Kutta (DIRK) methods, since they allow that the equations for each implicit stage are solved in a sequential manner. A class of Diagonally Implicit RungeKutta (DIRK) formulae, which are $A$ - stable and computationally efficient, was discussed in $[2,8,19]$. A special feature of the DIRK method in [8] is that it provides embedded DIRK formulae that allow a straightforward calculation of the local truncation error at each time step without extra computation. This provides an efficient way to estimate the time step required for a certain accuracy level. In [34] DIRK methods based on the minimization of certain error functions were considered, and several Butcher tables for specific DIRK formulae were given.

For the third order accurate LDG discretization in space, which uses quadratic basis functions, we use the third order Singly DIRK scheme from [34], given by

$$
\begin{aligned}
& \widehat{\mathbf{U}}^{n 1}=\widehat{\mathbf{U}}^{n}+\Delta t a_{11} \mathbf{K}_{1}, \quad \widehat{\mathbf{Z}}^{n 1}=\mathbf{P}\left(\widehat{\mathbf{U}}^{n 1}\right), \quad \mathbf{G}^{n 1}=\mathbf{Q}\left(\widehat{\mathbf{U}}^{n 1}, \widehat{\mathbf{Z}}^{n 1}\right), \\
& \mathbf{K}_{1}=-\mathbf{L}\left(t_{n}+c_{1} \Delta t, \widehat{\mathbf{U}}^{n 1}, \widehat{\mathbf{Z}}^{n 1}, \widehat{\mathbf{G}}^{n 1}\right), \\
& \widehat{\mathbf{U}}^{n 2}=\widehat{\mathbf{U}}^{n}+\Delta t a_{21} \mathbf{K}_{1}+\Delta t a_{22} \mathbf{K}_{2}, \quad \widehat{\mathbf{Z}}^{n 2}=\mathbf{P}\left(\widehat{\mathbf{U}}^{n 2}\right), \quad \widehat{\mathbf{G}}^{n 2}=\widehat{\mathbf{Q}}\left(\widehat{\mathbf{U}}^{n 2}, \widehat{\mathbf{Z}}^{n 2}\right), \\
& \mathbf{K}_{2}=-\mathbf{L}\left(t_{n}+c_{2} \Delta t, \widehat{\mathbf{U}}^{n 2}, \widehat{\mathbf{Z}}^{n 2}, \widehat{\mathbf{G}}^{n 2}\right), \\
& \widehat{\mathbf{U}}^{n 3}=\widehat{\mathbf{U}}^{n}+\Delta t a_{31} \mathbf{K}_{1}+\Delta t a_{32} \mathbf{K}_{2}+\Delta t a_{33} \mathbf{K}_{3}, \quad \widehat{\mathbf{Z}}^{n 3}=\mathbf{P}\left(\widehat{\mathbf{U}}^{n 3}\right), \quad \widehat{\mathbf{G}}^{n 3}=\mathbf{Q}\left(\widehat{\mathbf{U}}^{n 3}, \widehat{\mathbf{Z}}^{n 3}\right), \\
& \mathbf{K}_{3}=-\mathbf{L}\left(t_{n}+c_{3} \Delta t, \widehat{\mathbf{U}}^{n 3}, \widehat{\mathbf{Z}}^{n 3}, \widehat{\mathbf{G}}^{n 3}\right), \\
& \widehat{\mathbf{U}}^{n+1}=\widehat{\mathbf{U}}^{n}+\Delta t b_{1} \mathbf{K}_{1}+\Delta t b_{2} \mathbf{K}_{2}+\Delta t b_{3} \mathbf{K}_{3} .
\end{aligned}
$$

The coefficients in the SDIRK scheme are defined in the Butcher table

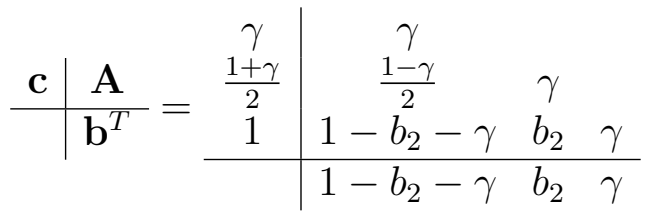

with $b_{2}=\frac{1}{4}\left(5-20 \gamma+6 \gamma^{2}\right)$ and $\gamma=0.43586652$. For the second order accurate LDG discretization in space, which uses linear polynomial basis functions, we 
use the second order accurate implicit Runge-Kutta time integration scheme

$$
\begin{aligned}
& \widehat{\mathbf{U}}^{n 1}=\widehat{\mathbf{U}}^{n}+\Delta t \frac{1}{2} \mathbf{K}_{1}, \quad \widehat{\mathbf{Z}}^{n 1}=\mathbf{P}\left(\widehat{\mathbf{U}}^{n 1}\right), \quad \widehat{\mathbf{G}}^{n 1}=\widehat{\mathbf{Q}}\left(\widehat{\mathbf{U}}^{n 1}, \widehat{\mathbf{Z}}^{n 1}\right), \\
& \mathbf{K}_{1}=-\mathbf{L}\left(t_{n}+\frac{1}{2} \Delta t, \widehat{\mathbf{U}}^{n 1}, \widehat{\mathbf{Z}}^{n 1}, \widehat{\mathbf{G}}^{n 1}\right), \\
& \widehat{\mathbf{U}}^{n+1}=\widehat{\mathbf{U}}^{n}+\frac{1}{2} \Delta t \mathbf{K}_{1} .
\end{aligned}
$$

Note that the intermediate stages $\mathbf{K}_{1}, \mathbf{K}_{2}$ and $\mathbf{K}_{3}$ must be solved implicitly, i.e. three (or one) large-scale nonlinear problems need to be dealt with. In recent years, great progress has been made in solving large nonlinear algebraic systems using globalized inexact Newton methods [15, 30]. The main difficulty with classical Newton methods is that a large-scale linear system needs to be solved at each iteration, which can be costly with a direct solver.

A popular alternative is a class of Newton-Krylov methods, which solve the large-scale nonlinear system iteratively. For more details, we refer to [25]. In the next section we describe the use of these methods to solve the nonlinear stage equations in the SDIRK method.

\subsection{Newton-Krylov methods}

Newton-Krylov methods solve the nonlinear equations

$$
F(x)=0, \quad \text { with } F \in \mathbb{R}^{N},
$$

in the following way: given an initial solution $x_{0}$, iteratively compute

$$
x_{k+1}=x_{k}+s, \quad \text { with } s \text { a solution of } D F\left(x_{k}\right) s=-F\left(x_{k}\right) \text {, }
$$

where $x_{k}$ is the current approximate solution and $D F\left(x_{k}\right)$ the Jacobian matrix of $F(x)$ at $x_{k}$. These methods combine outer nonlinear Newton iterations with inner linear Krylov iterations. The inner iteration stops when

$$
\left\|F\left(x_{k}\right)+D F\left(x_{k}\right) s_{k}\right\| \leq \eta_{k}\left\|F\left(x_{k}\right)\right\|
$$

where the constant $\eta_{k} \in(0,1)$ can be either fixed or specified dynamically.

We take the second order implicit Runge-Kutta time integration method (25) to explain the computation of the Jacobian matrix. System (25) can be 
rewritten as

$$
\begin{aligned}
& \mathbf{F}_{1} \triangleq \mathbf{K}_{1}+\mathbf{L}\left(t_{n}+\frac{1}{2} \Delta t, \widehat{\mathbf{U}}^{n 1}, \widehat{\mathbf{Z}}^{n 1}, \widehat{\mathbf{G}}^{n 1}\right)=0 \\
& \mathbf{F}_{2} \triangleq \widehat{\mathbf{Z}}^{n 1}-\mathbf{P}\left(\widehat{\mathbf{U}}^{n 1}\right)=0 \\
& \mathbf{F}_{3} \triangleq \widehat{\mathbf{G}}^{n 1}-\mathbf{Q}\left(\widehat{\mathbf{U}}^{n 1}, \widehat{\mathbf{Z}}^{n 1}\right)=0
\end{aligned}
$$

with $\widehat{\mathbf{U}}^{n 1}=\widehat{\mathbf{U}}^{n}+\Delta t \frac{1}{2} \mathbf{K}_{1}$. The Jacobian matrix $\mathbf{J}$ has the structure

$$
\mathbf{J}=\left(\begin{array}{ccc}
\frac{\partial \mathbf{F}_{1}}{\partial \mathbf{K}_{1}} & \frac{\partial \mathbf{F}_{1}}{\partial \widehat{\mathbf{Z}}^{n 1}} & \frac{\partial \mathbf{F}_{1}}{\partial \widehat{\mathbf{G}}^{n 1}} \\
\frac{\partial \mathbf{F}_{2}}{\partial \mathbf{K}_{1}} & \frac{\partial \mathbf{F}_{2}}{\partial \widehat{\mathbf{Z}}^{n 1}} & \mathbf{0} \\
\frac{\partial \mathbf{F}_{3}}{\partial \mathbf{K}_{1}} & \frac{\partial \mathbf{F}_{3}}{\partial \widehat{\mathbf{Z}}^{n 1}} & \frac{\partial \mathbf{F}_{3}}{\partial \widehat{\mathbf{G}}^{n 1}}
\end{array}\right)
$$

which is shown in Figure 2. It is not practical to solve the linear system in (27), with $D F\left(x_{k}\right)$ as Jacobian matrix, using a direct method. The linear system is therefore solved using GMRES with an ILU(0) preconditioner in order to prevent a large fill-in of the matrix.

\section{Numerical experiments}

In this section, we perform several numerical experiments to investigate the stability and accuracy of the LDG schemes for the (non)-isothermal NSK equations proposed in Sections 2.2 and 2.3. The examples in Section 4.2 are test cases for the isothermal NSK equations, including an investigation of the order of accuracy, a few one-dimensional benchmark problems and a two-dimensional simulation of the coalescence of two bubbles. These model problems were recently also (partly) studied in [29, 5] using a semi-discrete Galerkin method and a continuous finite element method, together with special time integration schemes. Compared to these methods the LDG schemes that we present in this article are relatively simple, robust and do not require additional regularization terms. The simulations of the non-isothermal NSK equations (6) are presented in Section 4.3, including accuracy verification, a static Riemann problem and a two-dimensional simulation of bubble coalescence. Note that we did not use any limiter in the computations. Periodic boundary conditions and a uniform mesh are applied for all test cases.

We use implicit Runge-Kutta(RK) time integration methods to solve the ODE system resulting from the LDG discretization for the accuracy tests 


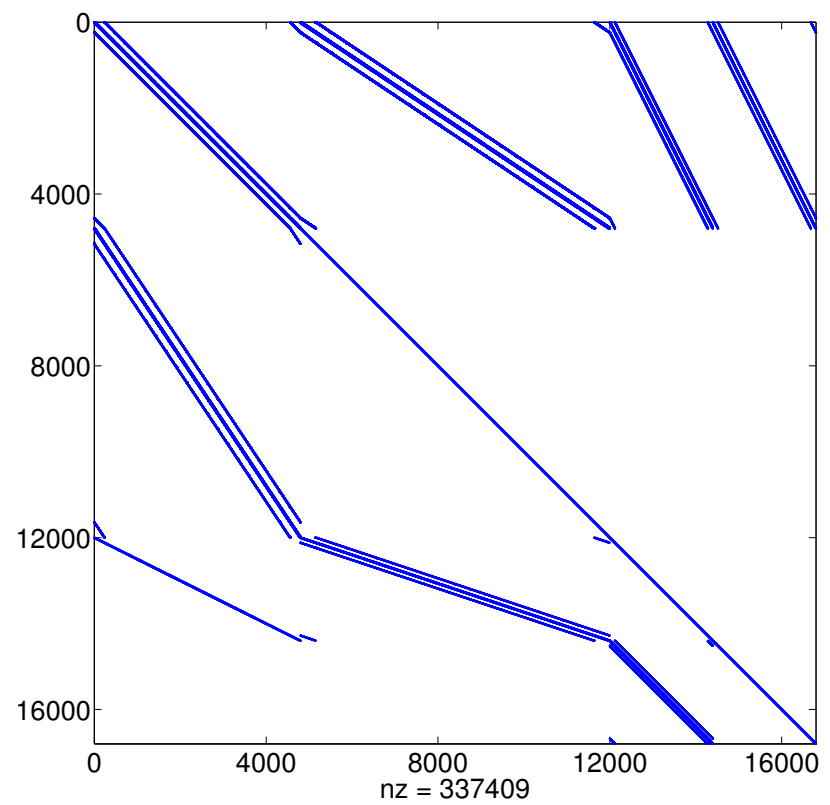

Figure 2: Non-zeros in the Jacobian matrix for the LDG discretization of the twodimensional non-isothermal NSK equations on a mesh with $20 \times 20$ elements.

and two dimensional bubble coalescence simulations. In the accuracy tests, the time step is chosen as $d t=0.8 h$ for the third order implicit RK time method, with $h$ the length of an element. The time step $d t=1.5 h$ is chosen for the second order implicit RK time method for the two dimensional bubble coalescence tests.

In several numerical examples, an equilibrium state with an interface between liquid and vapour is used to verify the capabilities of the proposed LDG scheme, described in Section 2.2. The velocity is denoted by $u$ in 1D, while $\mathbf{u}=(u, v)^{T}$ in 2D. At a certain dimensionless temperature $\theta<1$ in the Van der Waals equation of state, the densities in the equilibrium state $\rho_{v}, \rho_{l}$ that satisfy the following relations for the pressure and chemical potential

$$
\begin{aligned}
& p\left(\theta, \rho_{v}\right)=p\left(\theta, \rho_{l}\right), \\
& \mu\left(\theta, \rho_{v}\right)=\mu\left(\theta, \rho_{l}\right),
\end{aligned}
$$

are called Maxwell states. 


\subsection{Interface width}

To resolve the diffuse interface accurately, a sufficiently fine mesh is required in a simulation of a phase-field problem, otherwise the numerical solution will contain non-physical oscillations [29]. Suppose that the Helmholtz free energy is denoted by $f$, and that $\Delta f$ is the difference in Helmholtz free energy between the phase mixture and the separate phases:

$$
\Delta f=f-f_{0} .
$$

Here $f_{0}(\rho)=f\left(\rho_{v}, \theta_{0}\right)+\left(\rho-\rho_{v}\right) \frac{f\left(\rho_{l}, \theta_{0}\right)-f\left(\rho_{v}, \theta_{0}\right)}{\rho_{l}-\rho_{v}}$ for the given temperature $\theta_{0}$. The interface thickness [7] is then given by

$$
d=2 \frac{L}{\sqrt{\mathbb{W} e}} \frac{\rho_{l}-\rho_{v}}{\sqrt{\Delta f_{\max }}},
$$

with $\Delta f_{\max }$ the maximum value of $\Delta f, L$ the reference length scale and $\rho_{l}, \rho_{v}$ the critical densities at a given temperature. From the numerical tests, we found that at least 10 mesh nodes are required inside the interface to capture the interface accurately and guarantee the stability of the energy or entropy. This gives the relation $d=\alpha h, \alpha>10$ with $h$ the mesh size in the interface region.

\subsection{Numerical tests for the isothermal NSK equations}

Similar to [5, 29], choosing the temperature as $\theta=0.85$ and the Van der Waals equation of state (3), the critical vapour and liquid densities are equal to $\rho_{v}=0.106576655, \rho_{l}=0.602380109$.

\subsubsection{Accuracy test}

In this section, we will study the accuracy of the LDG discretization for the isothermal NSK equations. To investigate the accuracy of the onedimensional LDG discretization, we select an exact smooth solution as

$$
\begin{aligned}
& \rho=0.6+0.1 \sin (5 \pi t) \cos (2 \pi x), \\
& u=\sin (3 \pi t) \sin (2 \pi x),
\end{aligned}
$$

which satisfies (1) with an additional source term $\mathbf{S}$. The source terms are added artificially and obtained by inserting the chosen exact smooth solution (32) into (11). The computational domain is $\Omega=(0,1)$, and the coefficients in (1) are

$$
\mathbb{R} e=20, \quad \mathbb{W} e=100 \text {. }
$$


The solutions are obtained using the LDG discretization with piecewise linear and quadratic polynomials, combined with the third order implicit RungeKutta time integration method with stopping parameter $\eta_{k}$ in (28) chosen as $\eta_{k}=10^{-9}$. Table 1 shows the accuracy of the LDG scheme for the onedimensional isothermal NSK equations. From this table, we can see that the LDG discretizations have optimal order of accuracy for the different polynomial orders.

Table 1: Accuracy test of the LDG discretization for the one-dimensional isothermal NSK equations (1) with exact solution (30). The Van der Waals EOS is chosen as (3), $\theta=0.85$, and the physical parameters in the isothermal NSK equations (1) are set as (31). The LDG discretization uses linear and quadratic basis functions and periodic boundary conditions. Results are for uniform meshes with $M$ cells at time $t=0.1$.

\begin{tabular}{|c|c|cc|cc|}
\hline & $M$ & $\left\|\rho-\rho_{h}\right\|_{L^{2}(\Omega)}$ & order & $\left\|u-u_{h}\right\|_{L^{2}(\Omega)}$ & order \\
\hline \multirow{5}{*}{$P^{1}$} & 16 & $1.65 \mathrm{E}-03$ & - & $8.91 \mathrm{E}-03$ & - \\
& 32 & $4.06 \mathrm{E}-04$ & 2.03 & $2.27 \mathrm{E}-03$ & 1.97 \\
& 64 & $1.08 \mathrm{E}-05$ & 1.90 & $6.02 \mathrm{E}-04$ & 1.92 \\
& 128 & $2.83 \mathrm{E}-05$ & 1.94 & $1.56 \mathrm{E}-04$ & 1.95 \\
& 256 & $7.25 \mathrm{E}-06$ & 1.96 & $4.00 \mathrm{E}-05$ & 1.97 \\
\hline \multirow{5}{*}{$P^{2}$} & 16 & $1.21 \mathrm{E}-04$ & - & $7.20 \mathrm{E}-04$ & - \\
& 32 & $1.58 \mathrm{E}-05$ & 2.95 & $9.64 \mathrm{E}-05$ & 2.90 \\
& 64 & $2.18 \mathrm{E}-06$ & 2.86 & $1.30 \mathrm{E}-05$ & 2.90 \\
& 128 & $2.92 \mathrm{E}-07$ & 2.90 & $1.70 \mathrm{E}-06$ & 2.93 \\
& 256 & $3.81 \mathrm{E}-08$ & 2.94 & $2.18 \mathrm{E}-07$ & 2.96 \\
\hline
\end{tabular}

To investigate the accuracy of the LDG discretization for the two-dimensional isothermal NSK equations, we choose an exact smooth solution

$$
\begin{aligned}
& \rho=0.6+0.1 \sin (5 \pi t) \cos (2 \pi x) \cos (2 \pi y) \\
& u=\sin (3 \pi t) \sin (2 \pi x) \sin (2 \pi y) \\
& v=\sin (\pi t) \sin (4 \pi x) \sin (4 \pi y)
\end{aligned}
$$

which satisfies (11) with additional source terms. The computational domain is $\Omega=(0,1) \times(0,1)$ and square quadrilateral elements are used. Table 2 shows the results of the LDG scheme for the two-dimensional isothermal NSK equations using piecewise linear and quadratic polynomials, indicating that the LDG discretization in Section 2.2 has optimal order of accuracy. 
Table 2: Accuracy test of the LDG discretization for the two-dimensional isothermal NSK equations (11) with exact solution (32). The Van der Waals EOS is chosen as $(3), \theta=0.85$, and the physical parameters in the isothermal NSK equations (1) are set as (31). The LDG discretization uses piecewise linear and quadratic polynomials and periodic boundary conditions. Results are for uniform meshes with square elements at time $t=0.1$.

\begin{tabular}{|c|c|cc|cc|cc|}
\hline & Mesh & $\left\|\rho-\rho_{h}\right\|_{L_{2}}$ & order & $\left\|u-u_{h}\right\|_{L_{2}}$ & order & $\left\|v-v_{h}\right\|_{L_{2}}$ & order \\
\hline \multirow{5}{*}{$P^{1}$} & $16 \times 16$ & $1.82 \mathrm{E}-03$ & - & $3.95 \mathrm{E}-03$ & - & $1.53 \mathrm{E}-02$ & - \\
& $32 \times 32$ & $3.74 \mathrm{E}-04$ & 1.85 & $9.42 \mathrm{E}-04$ & 2.07 & $3.67 \mathrm{E}-03$ & 2.06 \\
& $64 \times 64$ & $1.49 \mathrm{E}-04$ & 1.95 & $2.36 \mathrm{E}-04$ & 2.00 & $9.08 \mathrm{E}-04$ & 2.01 \\
& $128 \times 128$ & $3.77 \mathrm{E}-05$ & 1.98 & $5.91 \mathrm{E}-05$ & 2.00 & $2.26 \mathrm{E}-04$ & 2.00 \\
\hline \multirow{5}{*}{$P^{2}$} & $16 \times 16$ & $3.38 \mathrm{E}-4$ & - & $3.08 \mathrm{E}-4$ & - & $1.81 \mathrm{E}-3$ & - \\
& $32 \times 32$ & $3.35 \mathrm{E}-5$ & 3.33 & $3.33 \mathrm{E}-5$ & 3.21 & $2.22 \mathrm{E}-4$ & 3.01 \\
& $64 \times 64$ & $3.85 \mathrm{E}-6$ & 3.12 & $3.89 \mathrm{E}-6$ & 3.10 & $2.81 \mathrm{E}-5$ & 3.00 \\
& $128 \times 128$ & $4.80 \mathrm{E}-7$ & 3.00 & $4.80 \mathrm{E}-7$ & 3.02 & $3.51 \mathrm{E}-6$ & 3.00 \\
\hline
\end{tabular}

\subsubsection{One-dimensional interface problem}

As a further verification of the accuracy and robustness of the LDG discretization, we solve two traveling wave problems for the one-dimensional isothermal NSK equations. It is known that some numerical discretizations produce solutions with overshoots, or incorrect wave speeds at discontinuities for this test case, see e.g. [29]. First, we consider a stationary wave problem with initial conditions

$$
\begin{gathered}
\rho_{0}(x)=\frac{\rho_{R}+\rho_{L}}{2}+\frac{\rho_{R}-\rho_{L}}{2} \tanh \left(\frac{x-0.5}{2} \sqrt{\mathbb{W} e}\right), \\
u_{0}(x)=\frac{u_{R}+u_{L}}{2}+\frac{u_{R}-u_{L}}{2} \tanh \left(\frac{x-0.5}{2} \sqrt{\mathbb{W} e}\right) .
\end{gathered}
$$

The coefficients in the initial conditions (33) are taken as

$$
\left(\rho_{L}, u_{L}\right)=(0.107,0), \quad\left(\rho_{R}, u_{R}\right)=(0.602,0) .
$$

We extend the domain $(0,1)$ to $[-1,1]$ with reflection symmetry at $x=0$ and use periodic boundary conditions. The physical parameters in (11) are set as $R e=200$ and $W e=10000$ and the mesh contains 400 elements. We plot the solutions of the LDG scheme for the isothermal NSK equations with piecewise linear and quadratic polynomials at time $t=0.1$ in Figure 3 . Figure 
3 shows that all numerical solutions are smooth without large oscillations, and the solutions resulting from linear and quadratic basis functions are indistinguishable at this mesh resolution. Since the initial condition is close to, but not exactly an equilibrium solution of the governing equations, the solution slightly changes in time and the velocity is small, but not equal to zero at the time shown in Figure 3 .

Next, we study a wave propagation problem. The initial conditions are set as (33) with

$$
\left(\rho_{R}, u_{R}\right)=(0.107,1.0), \quad\left(\rho_{L}, u_{L}\right)=(0.602,1.0)
$$

The LDG solution for the isothermal NSK equations with this initial condition results in a propagating traveling wave solution moving from the left to the right at speed 1.0. Again, stable numerical solutions are obtained as shown in Figure 4.

\subsubsection{Coalescence of two bubbles}

An important test case is the simulation of the coalescence of two bubbles, which was also studied in $[5,29]$. The computational domain is $[0,1]^{2}$. The parameters are $\mathbb{R} e=512, \mathbb{W} e=65500$. We consider two vapor bubbles of different radii, which are initially close to each other and at rest. The initial conditions are

$$
\rho_{0}(\mathbf{x})=\rho_{1}+\frac{1}{2}\left(\rho_{2}-\rho_{1}\right) \sum_{i=1}^{2} \tanh \left(\frac{\left(d_{i}(\mathbf{x})-r_{i}\right)}{2} \sqrt{\mathbb{W} e}\right), \quad \mathbf{u} \equiv \mathbf{0},
$$

with $\rho_{1}, \rho_{2}$ close to an equilibrium given a fixed constant temperature. Here $d_{i}(\mathbf{x})=\left\|\mathbf{x}-\mathbf{x}_{i}\right\|$ is the Euclidean distance and the points $\mathbf{x}_{i}$ are equal to $\mathbf{x}_{1}=(0.4,0.5)$ and $\mathbf{x}_{2}=(0.78,0.5)$, respectively. The radii of the two bubbles are $r_{1}=0.25$ and $r_{2}=0.1$. After some time, the bubbles will merge into one vapour bubble by capillarity and pressure forces.

Mass conservation and energy dissipation are critical parameters to investigate whether a numerical discretization for the NSK equations is suitable. To verify the mass conservation and energy dissipation properties of our LDG scheme, we denote the discrete mass and energy at time $t$, respectively, by

$$
\begin{aligned}
& m_{h}(t)=\int_{\Omega} \rho_{h}(t) d \mathbf{x} \\
& \mathcal{E}_{h}(t)=\int_{\Omega}\left(W\left(\rho_{h}(t)\right)+\frac{1}{2 \mathbb{W} e}\left|\nabla \rho_{h}(t)\right|^{2}+\frac{1}{2} \frac{\left|(\rho \mathbf{u})_{h}(t)\right|^{2}}{\rho_{h}(t)}\right) d \mathbf{x} .
\end{aligned}
$$




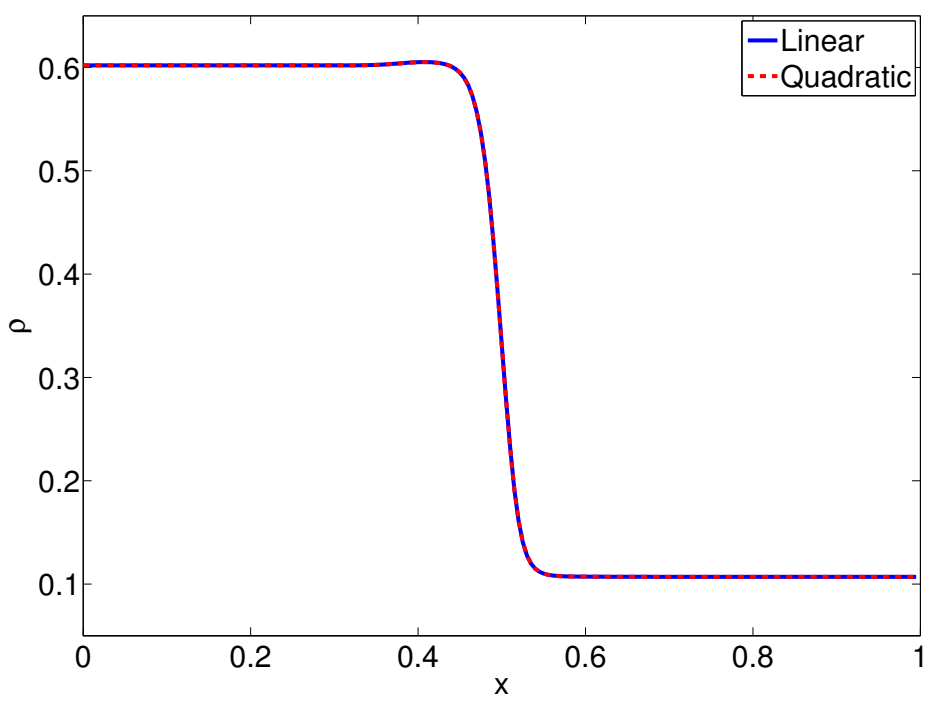

(a) density

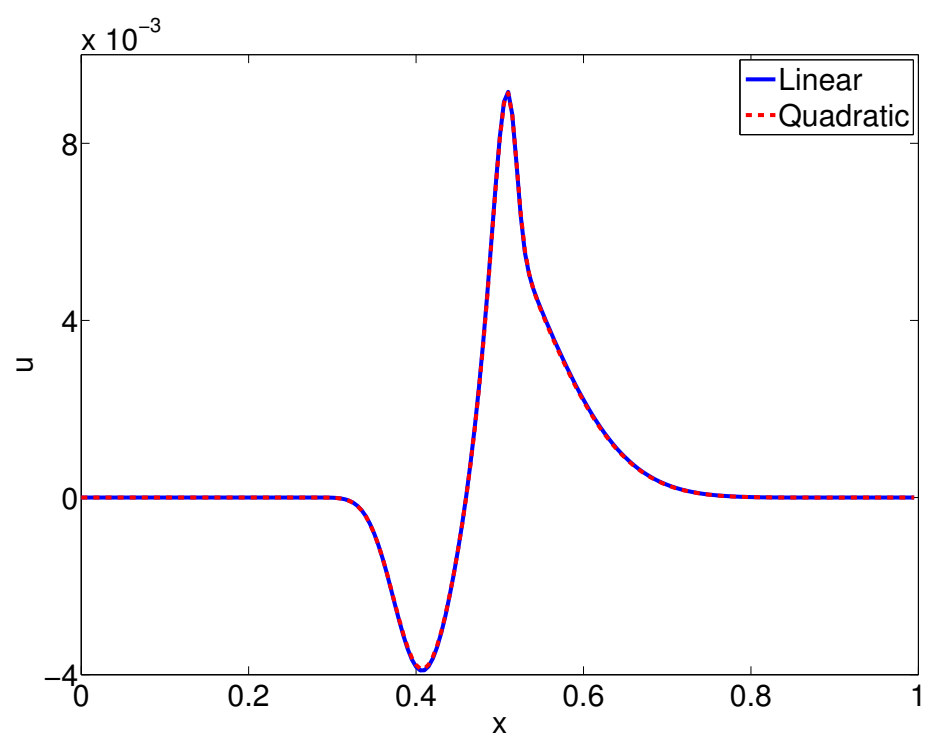

(b) velocity

Figure 3: One-dimensional stationary wave problem. LDG solutions with piecewise linear and quadratic polynomials at $t=0.1$ on a mesh containing 400 elements. The Van der Waals EOS is chosen as (3), $\theta=0.85$, and the physical parameters in the isothermal NSK equations (1) are set as $\mathbb{R e}=200$ and $\mathbb{W} e=10000$. 


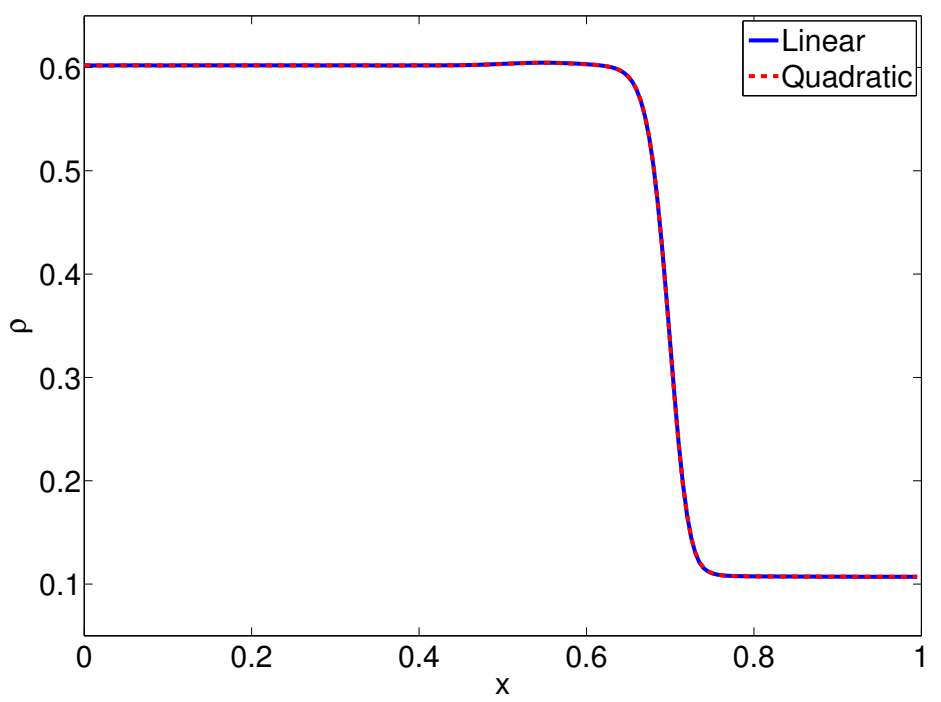

(a) density

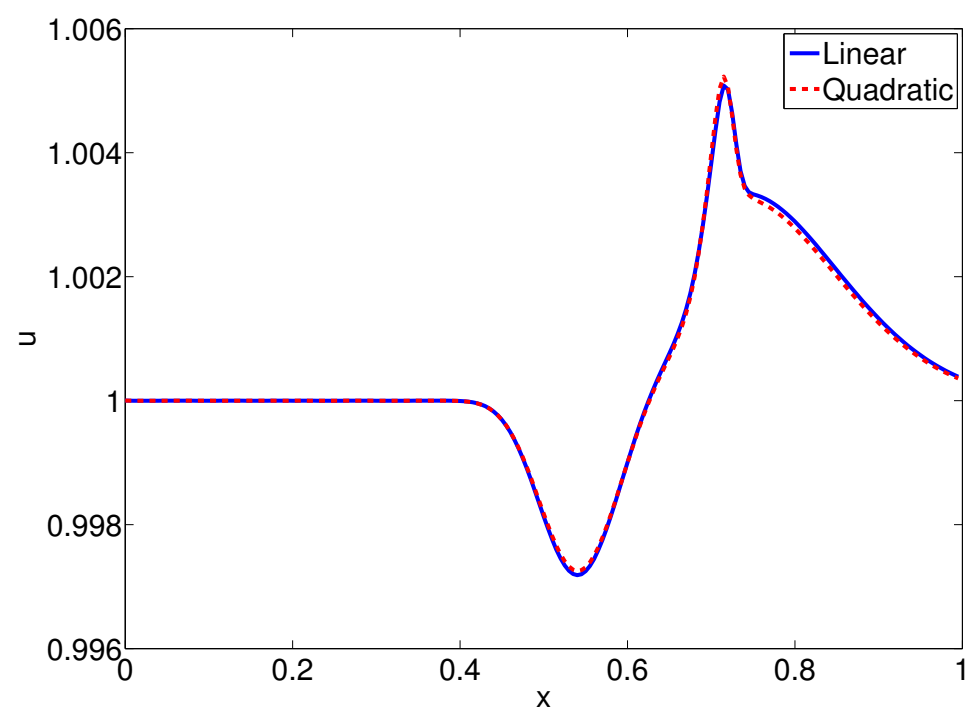

(b) velocity

Figure 4: One-dimensional propagating wave problem. LDG solutions with piecewise linear and quadratic polynomials at $t=0.2$ on a mesh containing 400 elements. The Van der Waals EOS is chosen as (3), $\theta=0.85$, and the physical parameters in the isothermal NSK equations (1) are set as $\mathbb{R} e=200$ and $\mathbb{W} e=10000$. 
The initial mass is $m_{h}\left(t_{0}\right)=\int_{\Omega} \rho_{0}(\mathbf{x}) d \mathbf{x}$.

Before using the LDG discretization, we will discuss the mesh required for this method to capture the interface and to represent the solution accurately. For the isothermal NSK equations with equation of state (3), (29), we obtain the following results:

- Given the temperature $\theta=0.85$, the critical densities are $\rho_{v}=0.107, \rho_{l}=$ 0.602. The interface width then is equal to

$$
d=\frac{2\left(\rho_{l}-\rho_{v}\right)}{\sqrt{\Delta f_{\max }}} \frac{1}{\sqrt{\mathbb{W} e}}=14.04 \frac{1}{\sqrt{\mathbb{W} e}} .
$$

Consequently $h=\frac{1}{\sqrt{\mathbb{W} e}}$ is a reasonable choice.

- Given the temperature $\theta=0.8$, the critical densities are $\rho_{v}=0.0800, \rho_{l}=$ 0.6442 . The interface width then is equal to

$$
d=\frac{2\left(\rho_{l}-\rho_{v}\right)}{\sqrt{\Delta f_{\max }}} \frac{1}{\sqrt{\mathbb{W} e}}=12.00 \frac{1}{\sqrt{\mathbb{W} e}} .
$$

Consequently $h=\frac{1}{\sqrt{W e}}$ is a reasonable choice also for this case.

We use the LDG discretization with piecewise linear and quadratic polynomials for the isothermal NSK equations and the second order implicit Runge-Kutta time integration method (25) with stopping parameter $\eta_{k}=$ $10^{-6}$ in the Newton method. Given $\theta=0.85$, the initial condition is set as (34) with $\rho_{1}=0.1, \rho_{2}=0.6$. We choose a mesh of $256^{2}$ square elements. Figure 5 presents the evolution for the mass loss and energy, showing that the mass is conserved, and the energy decreases monotonically in time. The evolution of the bubble coalescence process is shown in Figures 6 and 7. These figures show that the two bubbles, which are below the critical temperature and initially close to each other and at rest, merge into one bubble during the simulation because of surface tension. After coalescence the resulting bubble slowly reaches an equilibrium state, in which the interface has a constant radius of curvature due to surface tension and the velocity field approaches zero.

The method for the isothermal NSK equations with $\theta=0.85$ was also tested on a coarser mesh with $128^{2}$ elements. Figure 8 shows the density profiles at various times, indicating that stable results are still obtained on this coarser mesh. The density and pressure along the line $y=0.5$ are 


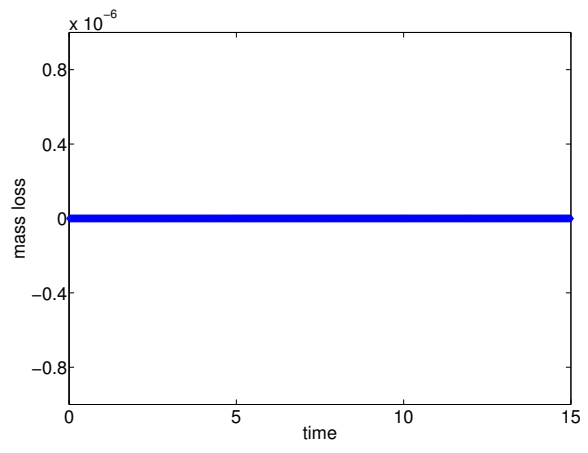

(a) mass loss

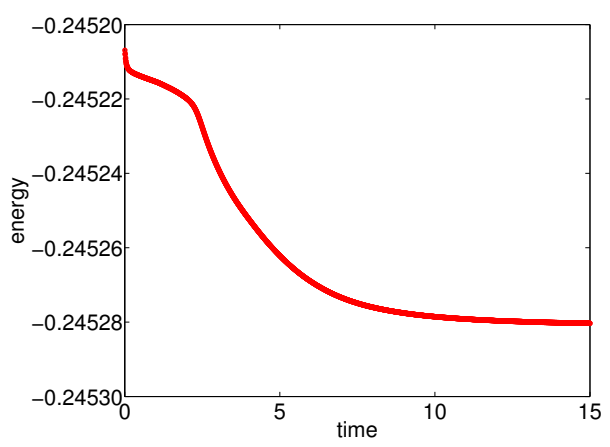

(b) energy

Figure 5: Evolution of mass loss and energy as a function of time during the coalescence of two bubbles computed with the LDG discretization of the isothermal NSK equations using piecewise linear polynomials on a mesh with $256^{2}$ square elements. The Van der Waals EOS is chosen as (3), $\theta=0.85$, and the physical parameters in the isothermal NSK equations (1) are set as $\mathbb{R} e=512, \mathbb{W} e=65500$. The initial condition is (34) with $\rho_{1}=0.1, \rho_{2}=0.6$.

displayed in Figure 9, which shows that the diffuse interface has only 8 nodes in this test. The evolution of the energy is displayed in Figure 10, showing that the energy is slightly increasing on a mesh with $128^{2}$ elements for the isothermal NSK equations with $\mathbb{W} e=65500$. On meshes coarser with less than 8 nodes in the diffuse interface no stable results are obtained for this Weber number.

Choosing $\theta=0.8,(29)$ results in critical densities $\rho_{l}=0.6442, \rho_{v}=0.0800$ with a larger density ratio. When the LDG discretization is applied to the isothermal NSK equations with $\theta=0.8$ and initial condition (34) with $\rho_{1}=$ $0.08, \rho_{2}=0.64$, stable results are still obtained on a mesh of $256^{2}$ elements, as can be seen from Figure 12. Figure 11 shows that the mass is conserved, and the energy is only slightly increasing in time.

We also study the behaviour of the numerical scheme for the isothermal NSK equations when the initial densities are further away from the equilibrium densities $\rho_{l}, \rho_{v}$. For example, given a fixed temperature $\theta=0.85$, $\rho_{1}=0.05, \rho_{2}=0.65$ were chosen in (34). We choose the parameters in (1) as $\mathbb{R} e=500, \mathbb{W} e=10000$, and the mesh of $100^{2}$ elements. Mass and energy properties are presented in figure 13, which shows that the mass is conserved and the energy is decreasing in time. Figure 14 shows the coalescence of 


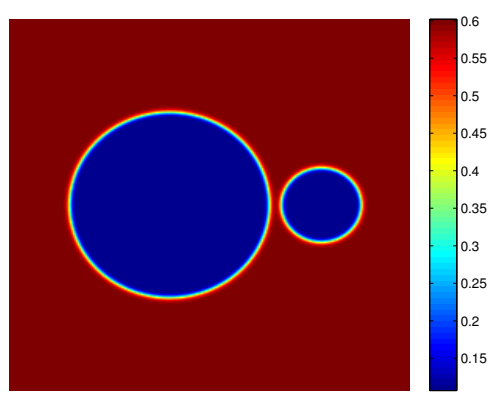

(a) time $t=0$.

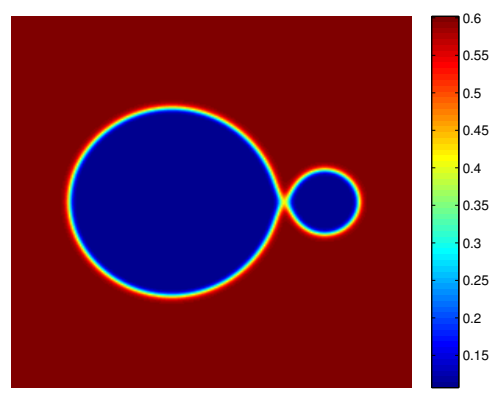

(c) time $t=2.0$.

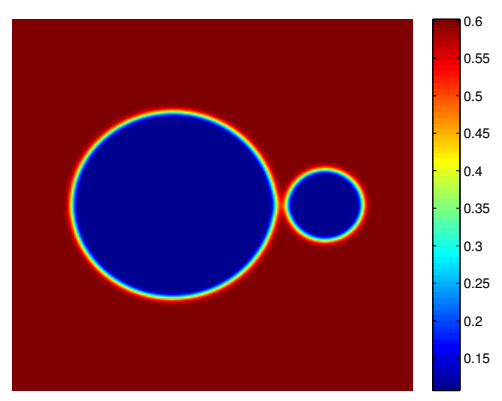

(b) time $t=1.0$.

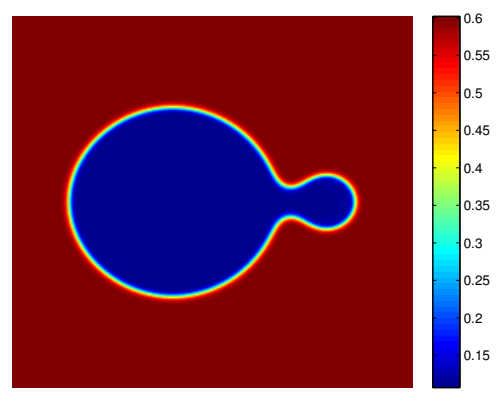

(d) time $t=3.0$.

Figure 6: Density $\rho$ for two coalescing bubbles computed with the LDG discretization of the isothermal NSK equations using piecewise linear polynomials on a mesh with $256^{2}$ square elements. The Van der Waals EOS is chosen as (3), $\theta=0.85$, and the physical parameters in the isothermal NSK equations (1) are set as $\mathbb{R} e=512$, We $=65500$. The initial condition is (34) with $\rho_{1}=0.1, \rho_{2}=0.6$. 


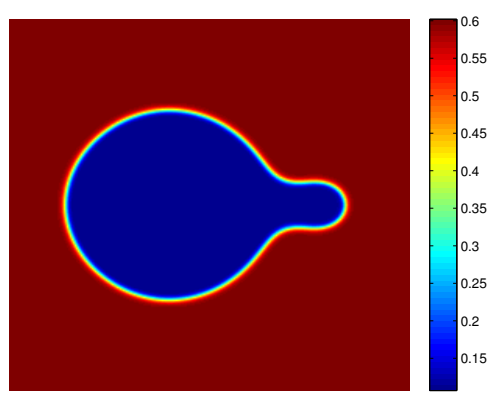

(a) time $t=4.0$.

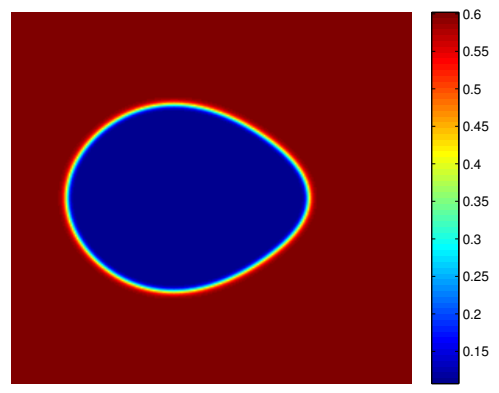

(c) time $t=9.0$.

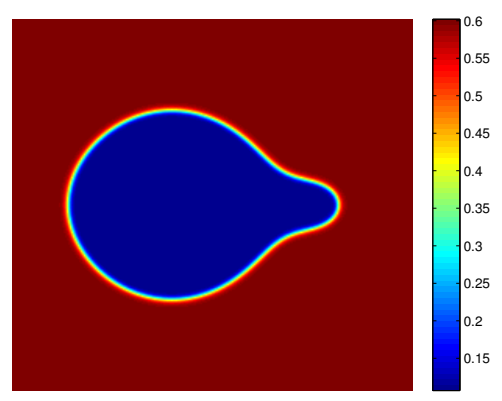

(b) time $t=5.0$.

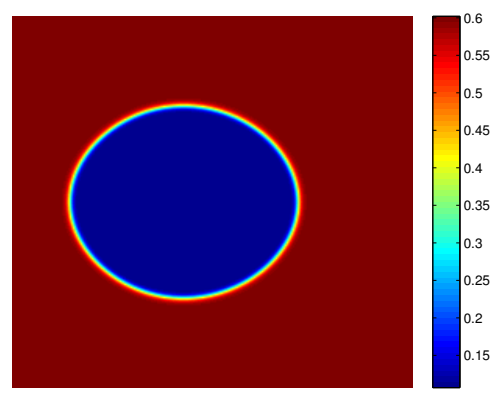

(d) time $t=15.0$.

Figure 7: Density $\rho$ for two coalescing bubbles computed with the LDG discretization of the isothermal NSK equations using piecewise linear polynomials on a mesh with $256^{2}$ square elements. The Van der Waals EOS is chosen as (3), $\theta=0.85$, and the physical parameters in the isothermal NSK equations (1) are set as $\mathbb{R} e=512$, $\mathbb{W} e=65500$. The initial condition is (34) with $\rho_{1}=0.1, \rho_{2}=0.6$. 


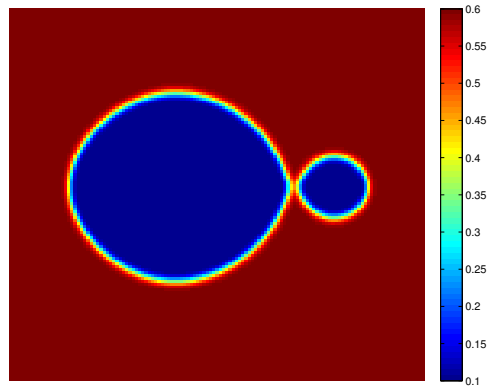

(a) $\mathrm{t}=2.0$

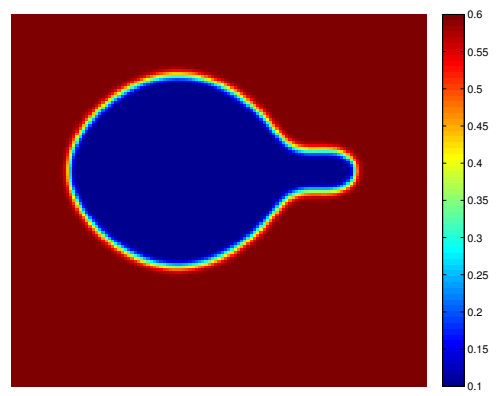

(c) $\mathrm{t}=4.0$

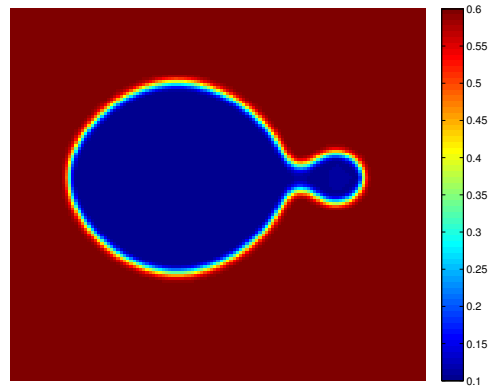

(b) $\mathrm{t}=3.0$

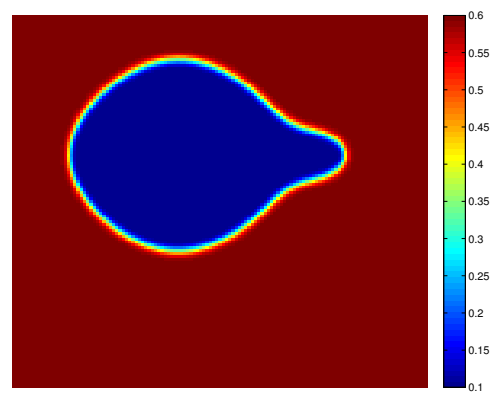

(d) $\mathrm{t}=5.0$

Figure 8: Density $\rho$ for two coalescing bubbles computed with the LDG discretization of the isothermal NSK equations using piecewise linear polynomials on a mesh with $128^{2}$ square elements. The Van der Waals EOS is chosen as (3), $\theta=0.85$, and the physical parameters in the isothermal NSK equations (1) are set as $\mathbb{R} e=512$, We $=65500$. The initial condition is (34) with $\rho_{1}=0.1, \rho_{2}=0.6$. 


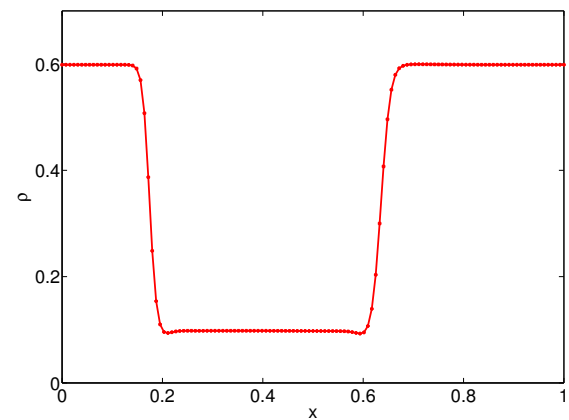

(a) density along $y=0.5$

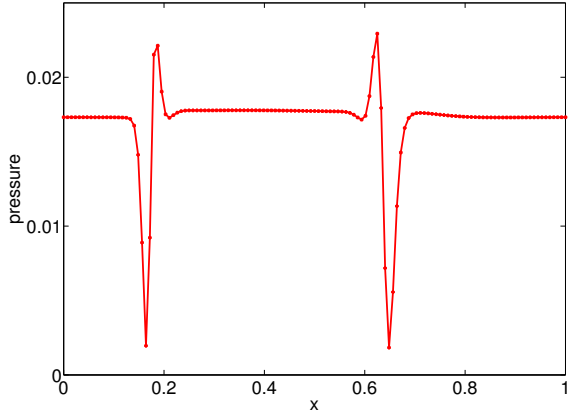

(b) pressure along $y=0.5$

Figure 9: Density and pressure along $y=0.5$ at time $t=5.0$ for the coalescence of two bubbles for the isothermal NSK equations using piecewise linear polynomials on a mesh with $128^{2}$ square elements.The Van der Waals EOS is chosen as (3), $\theta=0.85$, and the physical parameters in the isothermal NSK equations (1) are set as $\mathbb{R} e=512, \mathbb{W} e=65500$. The initial condition is (34) with $\rho_{1}=0.1, \rho_{2}=0.6$.

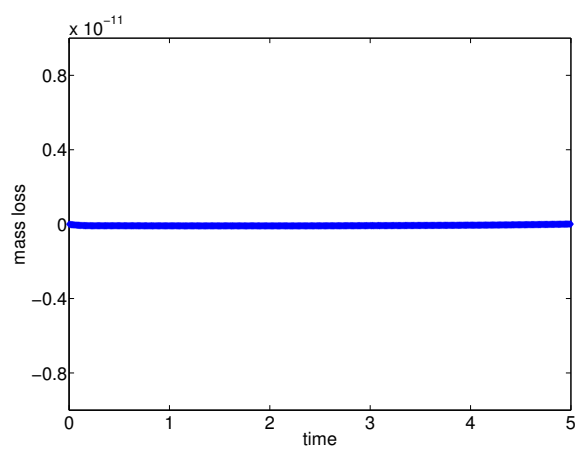

(a) mass loss

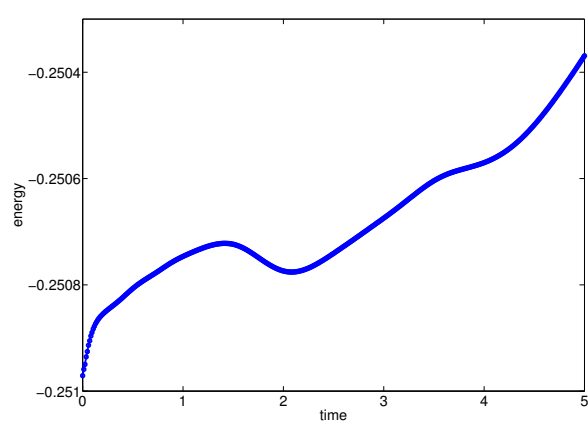

(b) energy

Figure 10: Evolution of mass loss and energy as a function of time during the coalescence of two bubbles computed with the LDG discretization of the isothermal NSK equations using piecewise linear polynomials on a mesh with $128^{2}$ square elements. The Van der Waals EOS is chosen as (3), $\theta=0.85$ and the physical parameters in the isothermal NSK equations (1) are set as $\mathbb{R} e=512$, We $e=65500$. The initial condition is (34) with $\rho_{1}=0.1, \rho_{2}=0.6$. 


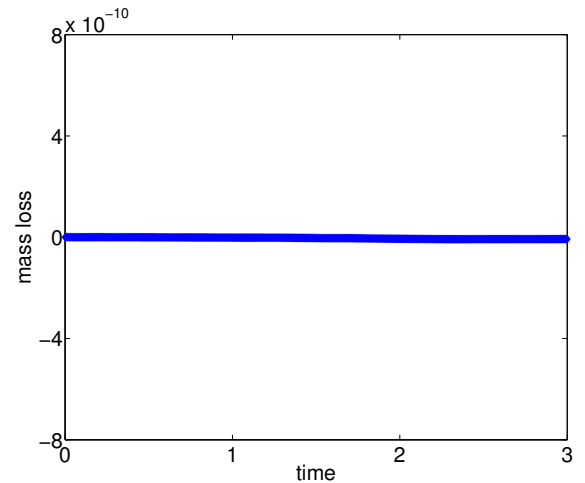

(a) mass loss

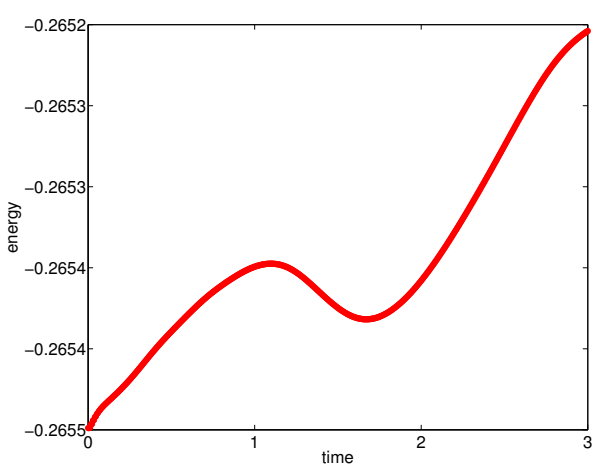

(b) energy

Figure 11: Evolution of mass loss and energy as a function of time during the simulation of two bubbles for the isothermal NSK equations with $\mathbb{R} e=512$, $\mathbb{W} e=65500$ on a mesh of $256^{2}$ elements using piecewise linear polynomials. The Van der Waals EOS is chosen as (3), $\theta=0.8$. The initial condition is (34) with $\rho_{1}=0.08, \rho_{2}=0.64$.

bubbles for the isothermal NSK equations with $R e=200, W e=10000$ when the initial density is far away from an equilibrium on a mesh of $100^{2}$ elements. Figure 15 shows the results for the isothermal NSK equations with $R e=512, W e=65500$ when the initial density is far away from equilibrium on a mesh of $256^{2}$ elements. Because of the non-equilibrium initial condition we get sound waves traveling to the boundaries of the domain and transported back into the domain on the opposite side due to the periodic boundary conditions. For the isothermal NSK equations with $R e=512, W e=65500$ on a mesh of $256^{2}$ elements, the amplitude of these sound waves is so large that this results in regions where the density is so low that a bubble occurs there. Since the simulations are isothermal, there is no latent heat that prevents this. The large Weber number also helps the formation of a bubble, since the surface tension is rather low.

\subsection{Numerical experiments for the non-isothermal NSK equations}

Choosing the temperature $\theta=0.989$ andthe Van der Waals equation of state (8), the Maxwell states are

$$
\rho_{v}=0.79525689, \quad \rho_{l}=1.21357862
$$




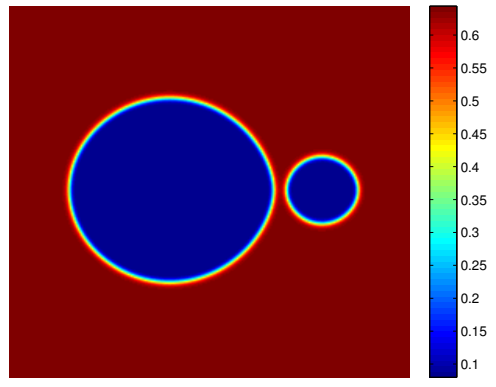

(a) time $t=1$.

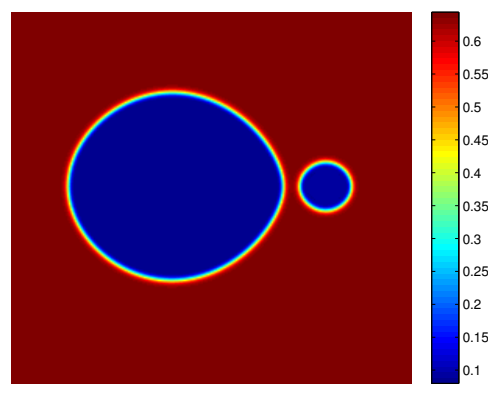

(c) time $t=2.5$.

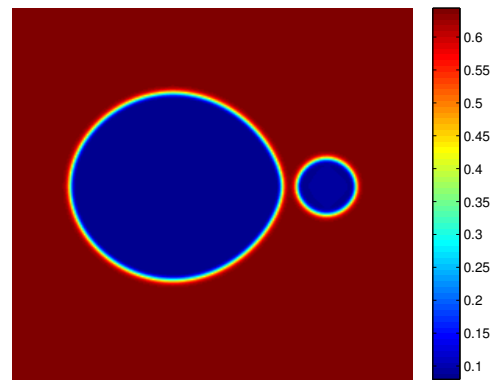

(b) time $t=2$.

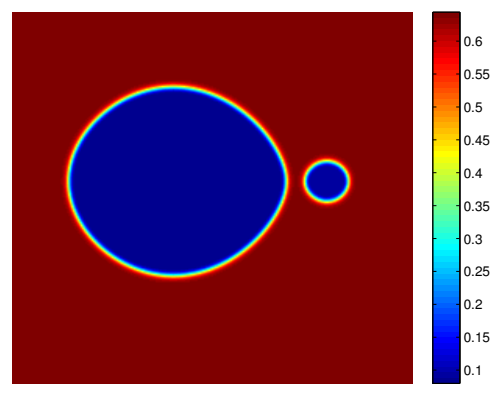

(d) time $t=3$.

Figure 12: Density $\rho$ for two bubbles computed with the LDG discretization of the isothermal NSK equations with $\mathbb{R} e=512$, We $=65500$ on a mesh of $256^{2}$ elements using piecewise linear polynomials. The Van der Waals EOS is chosen as (3), $\theta=0.8$. The initial condition is (34) with $\rho_{1}=0.08, \rho_{2}=0.64$. 


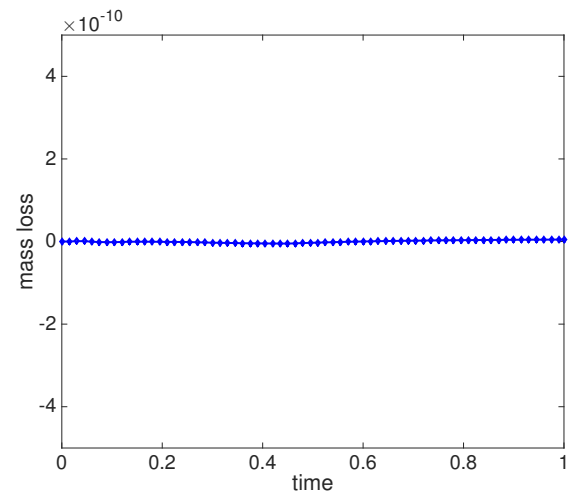

(a) mass loss

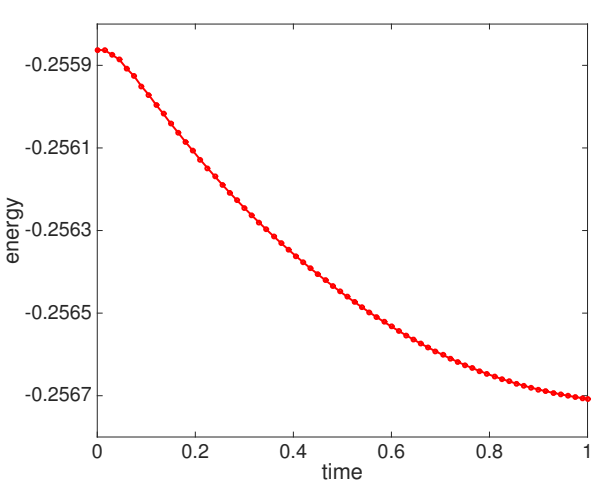

(b) energy

Figure 13: Evolution of mass loss and energy as a function of time during the coalescence of two bubbles computed with the LDG discretization of the isothermal NSK equations with $\mathbb{R} e=200, \mathbb{W} e=10000$ on a mesh with $100^{2}$ square elements. Piecewise linear polynomials are used. The Van der Waals EOS is chosen as (3), $\theta=0.85$. The initial condition is (34) with $\rho_{1}=0.05, \rho_{2}=0.65$.

\subsubsection{Accuracy test}

For the accuracy test of the LDG discretization of the one-dimensional non-isothermal NSK equations (6), a smooth exact solution is chosen as

$$
\left\{\begin{array}{l}
\rho(x, t)=0.6+0.1 \sin (5 \pi t) \cos (2 \pi x), \\
v(x, t)=\sin (3 \pi t) \sin (2 \pi x), \\
\theta(x, t)=0.8+0.1 \sin (\pi t) \sin (2 \pi x),
\end{array}\right.
$$

which satisfies the non-isothermal NSK equations (6) with a properly chosen source term $\mathbf{S}$. The Prandtl number $\mathbb{P r}$ in (6) is chosen as $\mathbb{P r}=0.843$, and $C_{v}=5.375$.

In order to verify the accuracy of the LDG scheme for the two-dimensional 


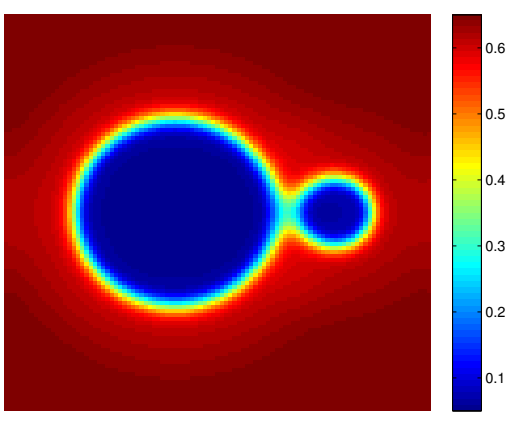

(a) $\mathrm{t}=0.2$

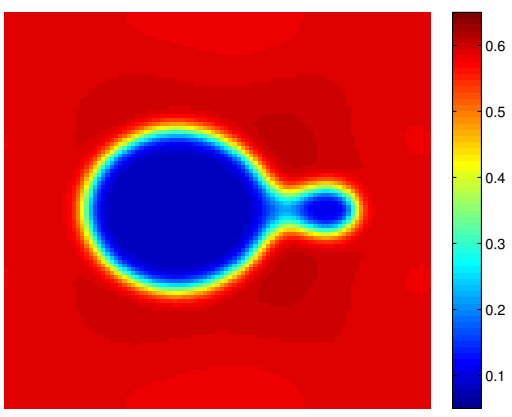

(c) $\mathrm{t}=0.6$

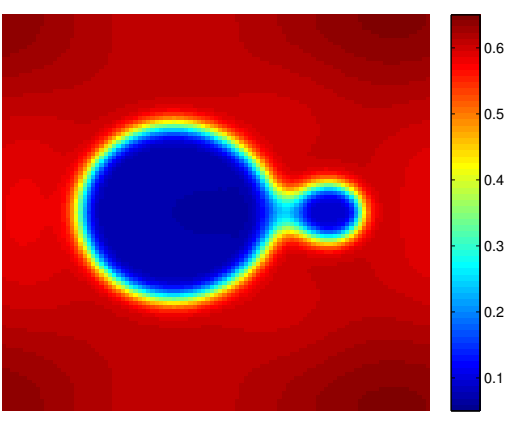

(b) $\mathrm{t}=0.4$

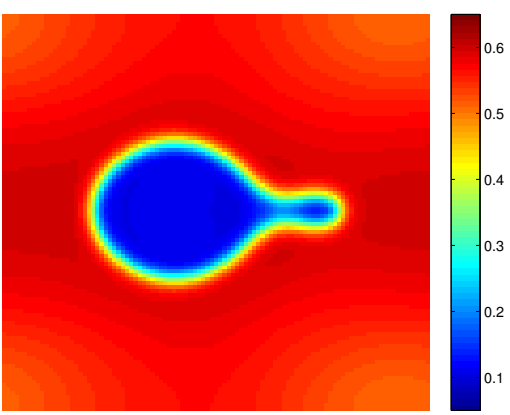

(d) $\mathrm{t}=1.0$

Figure 14: Coalescence of two bubbles for the isothermal NSK equations with $\mathbb{R} e=$ $200, \mathbb{W} e=10000$ on a mesh of $100^{2}$ elements. Piecewise linear polynomials are used. The Van der Waals EOS is chosen as (3), $\theta=0.85$. The initial condition is (34) with $\rho_{1}=0.05, \rho_{2}=0.65$. 


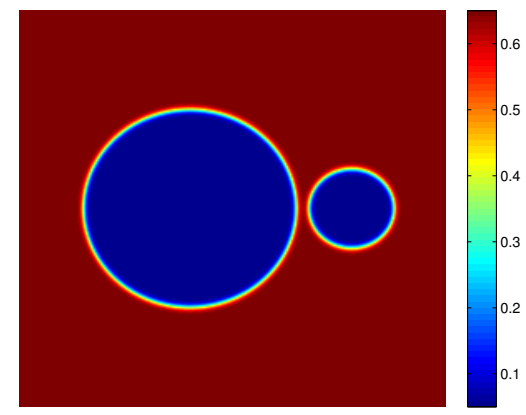

(a) $\mathrm{t}=0$

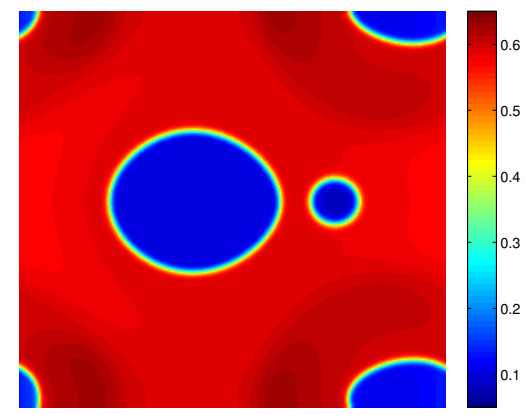

(c) $t=2$

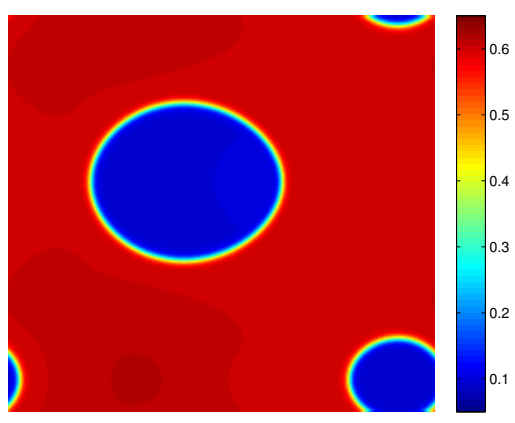

(e) $t=4$

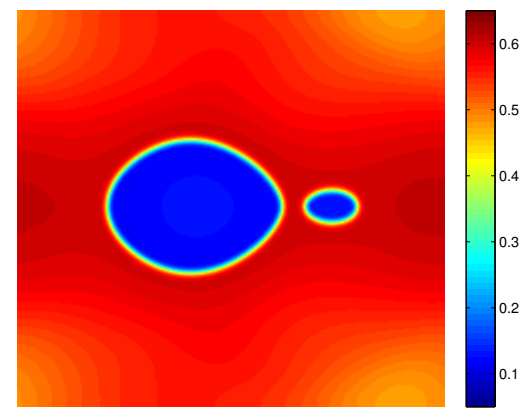

(b) $t=1$

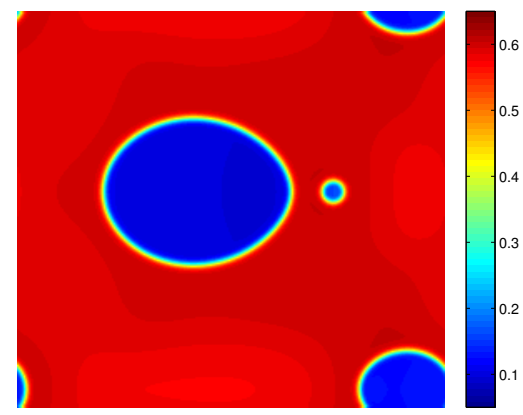

(d) $\mathrm{t}=3$

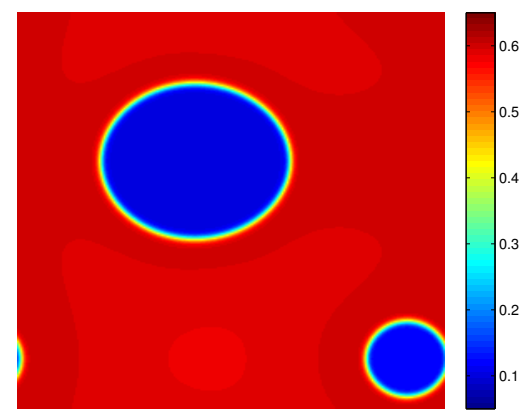

(f) $t=5$

Figure 15: Coalescence of two bubbles for the isothermal NSK equations with $\mathbb{R} e=$ $512, \mathbb{W} e=65500$ on a mesh of $256^{2}$ elements. Piecewise linear polynomials are used. The Van der Waals EOS is chosen as (3), $\theta=0.85$. The initial condition is (34) with $\rho_{1}=0.05, \rho_{2}=0.65$. 
Table 3: Accuracy test of the LDG discretization for the one-dimensional non-isothermal NSK equations (6) with exact solution (36). The physical parameters are chosen as $R e=$ $50, W e=1000, \operatorname{Pr}=0.843, C_{v}=5.375$, and the Van der Waals EOS is set as (8). LDG discretization with piecewise linear and quadratic polynomials, periodic boundary conditions, uniform meshes and time $t=0.1$.

\begin{tabular}{|c|c|cc|cc|cc|}
\hline & Mesh & $\left\|\rho-\rho_{h}\right\|_{L_{2}}$ & order & $\left\|u-u_{h}\right\|_{L_{2}}$ & order & $\left\|\theta-\theta_{h}\right\|_{L_{2}}$ & order \\
\hline \multirow{5}{*}{$P^{1}$} & 16 & $1.43 \mathrm{E}-03$ & - & $3.65 \mathrm{E}-03$ & - & $2.88 \mathrm{E}-04$ & - \\
& 32 & $3.551 \mathrm{E}-04$ & 2.01 & $9.32 \mathrm{E}-04$ & 1.97 & $7.44 \mathrm{E}-05$ & 1.95 \\
& 64 & $8.89 \mathrm{E}-05$ & 2.00 & $2.36 \mathrm{E}-04$ & 1.98 & $1.90 \mathrm{E}-05$ & 1.97 \\
& 128 & $2.22 \mathrm{E}-05$ & 2.00 & $5.96 \mathrm{E}-05$ & 1.99 & $4.80 \mathrm{E}-06$ & 1.99 \\
& 256 & $5.56 \mathrm{E}-06$ & 2.00 & $1.50 \mathrm{E}-05$ & 1.99 & $1.20 \mathrm{E}-06$ & 2.00 \\
\hline \multirow{5}{*}{$P^{2}$} & 16 & $2.71 \mathrm{E}-5$ & - & $1.35 \mathrm{E}-4$ & - & $1.21 \mathrm{E}-5$ & - \\
& 32 & $2.14 \mathrm{E}-6$ & 3.66 & $1.71 \mathrm{E}-5$ & 2.68 & $1.33 \mathrm{E}-6$ & 3.18 \\
& 64 & $2.42 \mathrm{E}-7$ & 3.14 & $2.18 \mathrm{E}-6$ & 2.97 & $1.54 \mathrm{E}-7$ & 3.11 \\
& 128 & $2.96 \mathrm{E}-8$ & 3.03 & $2.77 \mathrm{E}-7$ & 2.98 & $1.86 \mathrm{E}-8$ & 3.04 \\
& 256 & $3.70 \mathrm{E}-9$ & 3.00 & $3.50 \mathrm{E}-8$ & 2.96 & $2.31 \mathrm{E}-9$ & 3.01 \\
\hline
\end{tabular}

non-isothermal NSK equations (6), we select the exact smooth solution

$$
\left\{\begin{array}{l}
\rho(x, t)=0.6+0.1 \sin (5 \pi t) \cos (2 \pi x) \cos (2 \pi y), \\
u(x, t)=\sin (3 \pi t) \sin (2 \pi x) \sin (2 \pi y), \\
v(x, t)=\sin (3 \pi t) \sin (4 \pi x) \sin (4 \pi y), \\
\theta(x, t)=0.8+0.1 \sin (\pi t) \sin (2 \pi x) \cos (2 \pi y),
\end{array}\right.
$$

which satisfies the non-isothermal NSK equations (6) with a properly chosen source term. The results of the accuracy tests of the LDG discretization for the 1D and 2D non-isothermal NSK equations are given in Tables 3 and 4, respectively. From these results, we can see that the LDG discretization for the non-isothermal NSK equations has optimal order of accuracy.

\subsubsection{One-dimensional interface problem}

In this section we consider a one-dimensional interface problem to investigate the accuracy and robustness of the LDG discretization of the nonisothermal NSK equations. The initial conditions are set as a similar form 
Table 4: Accuracy test of the LDG discretization for the two-dimensional non-isothermal NSK equations (6) with exact solution (37). The physical parameters are chosen as $R e=$ $50, W e=1000, \operatorname{Pr}=0.843, C_{v}=5.375$, and the Van der Waals EOS is set as (8). LDG discretization with piecewise linear and quadratic polynomials, periodic boundary conditions, and uniform meshes with square elements at time $t=0.1$.

\begin{tabular}{|c|c|cc|cc|cc|}
\hline & Mesh & $\left\|\rho-\rho_{h}\right\|_{L_{2}}$ & order & $\left\|u-u_{h}\right\|_{L_{2}}$ & order & $\left\|\theta-\theta_{h}\right\|_{L_{2}}$ & order \\
\hline \multirow{5}{*}{$P^{1}$} & $16 \times 16$ & $2.08 \mathrm{E}-03$ & - & $3.72 \mathrm{E}-02$ & - & $9.85 \mathrm{E}-4$ & - \\
& $32 \times 32$ & $5.61 \mathrm{E}-04$ & 1.88 & $9.36 \mathrm{E}-04$ & 1.99 & $2.63 \mathrm{E}-4$ & 1.90 \\
& $64 \times 64$ & $1.47 \mathrm{E}-04$ & 1.94 & $2.34 \mathrm{E}-04$ & 2.00 & $6.91 \mathrm{E}-5$ & 1.94 \\
& $128 \times 128$ & $3.76 \mathrm{E}-05$ & 1.97 & $5.85 \mathrm{E}-05$ & 2.00 & $1.76 \mathrm{E}-5$ & 1.97 \\
\hline \multirow{4}{*}{$P^{2}$} & $16 \times 16$ & $5.11 \mathrm{E}-4$ & - & $6.66 \mathrm{E}-4$ & - & $7.36 \mathrm{E}-4$ & - \\
& $32 \times 32$ & $5.57 \mathrm{E}-5$ & 3.19 & $6.94 \mathrm{E}-5$ & 3.21 & $1.28 \mathrm{E}-4$ & 2.51 \\
& $64 \times 64$ & $6.93 \mathrm{E}-6$ & 3.01 & $7.99 \mathrm{E}-6$ & 3.11 & $1.56 \mathrm{E}-5$ & 3.04 \\
& $128 \times 128$ & $8.74 \mathrm{E}-7$ & 2.98 & $9.70 \mathrm{E}-7$ & 3.04 & $1.78 \mathrm{E}-6$ & 3.13 \\
\hline
\end{tabular}

as (33) with

$$
\left(\rho_{L}, u_{L}, \theta_{L}\right)=(0.795,0.0,0.989), \quad\left(\rho_{R}, u_{R}, \theta_{R}\right)=(1.213,0.0,0.989) .
$$

The domain is $[-5,5]$.

Figure 16 shows that the LDG scheme for the non-isothermal NSK equations results in accurate and stable solutions. Figure 17 compares the LDG solutions for the isothermal and non-isothermal NSK equations with the Van der Waals equation of state (8). The dimensionless numbers are equal to $\mathbb{R} e=128.6, \mathbb{W} e=968.6$ and the initial conditions are defined in (38). Figure 17 shows that, compared to the isothermal NSK equations, the LDG solutions for the non-isothermal NSK equations result in less oscillations in the density near the interface.

\subsubsection{Coalescence of two bubbles}

Next, we simulate the coalescence of two bubbles. The parameters are $\mathbb{R} e=950, \mathbb{W} e=34455$. The computational domain is $[0,1]^{2}$. We consider two vapor bubbles of different radii, which are initially close to each other and at rest. The initial conditions are

$$
\rho_{0}(\mathbf{x})=\rho_{1}+\frac{1}{2}\left(\rho_{2}-\rho_{1}\right) \sum_{i=1}^{2} \tanh \left(\frac{\left(d_{i}(\mathbf{x})-r_{i}\right)}{2} \sqrt{\mathbb{W} e}\right), \quad \mathbf{u} \equiv \mathbf{0}, \quad \theta=\theta_{0}
$$




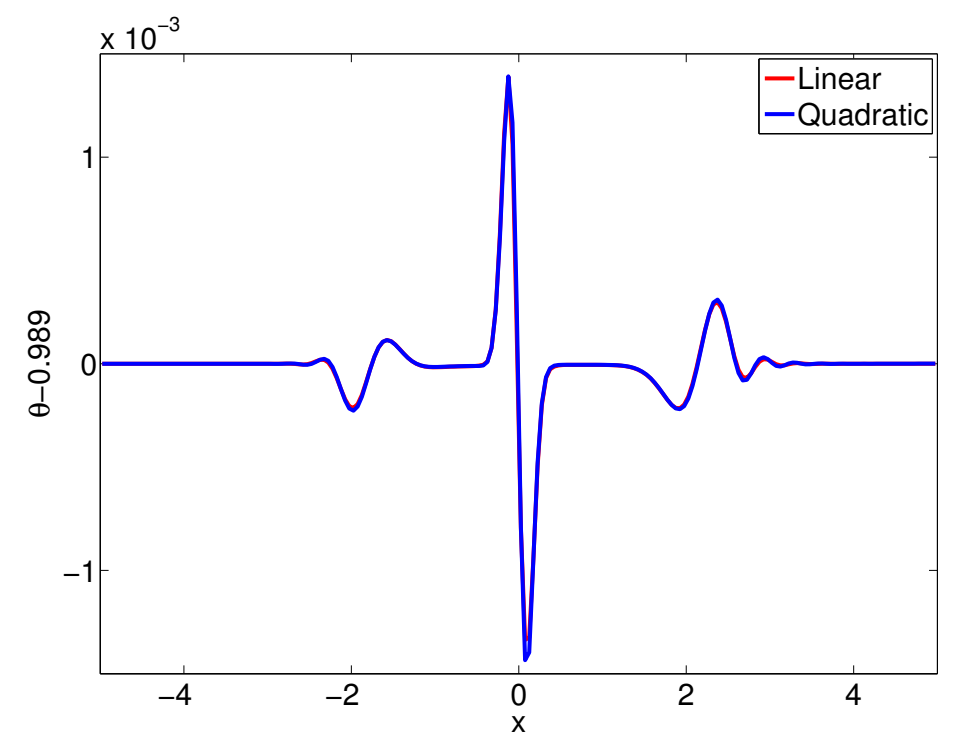

(a) $\theta-0.989$

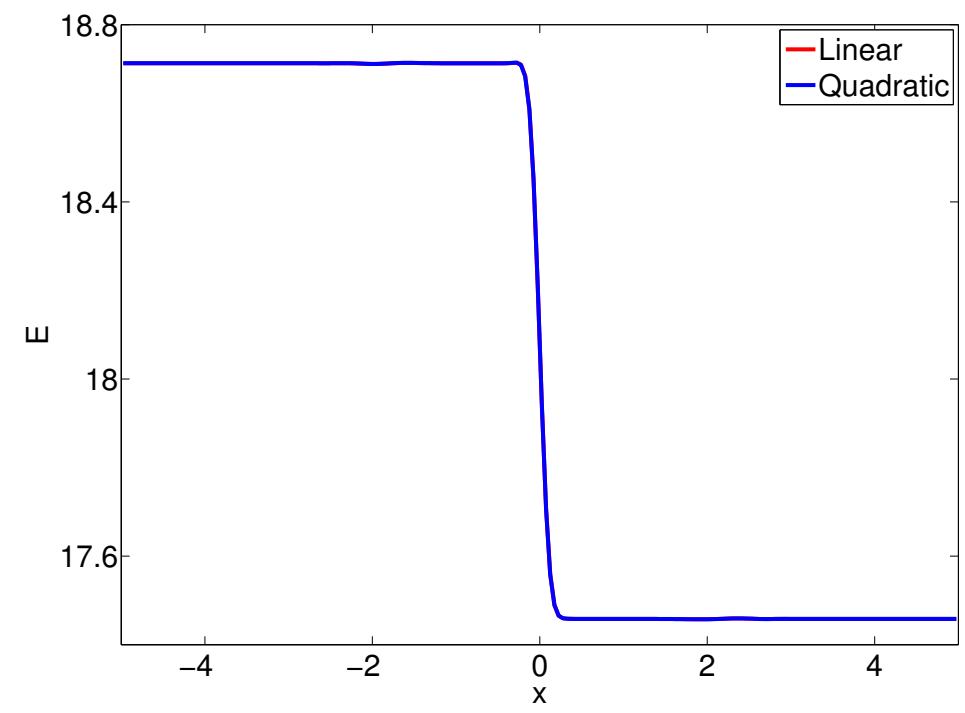

(b) Energy

Figure 16: One-dimensional static interface problem for the non-isothermal NSK equations. Numerical solutions obtained with the LDG scheme with piecewise quadratic polynomials at $t=2.0$ on a mesh containing 400 elements. The physical parameters are chosen as $\mathbb{R} e=128.6, \mathbb{W} e=968.6, \mathbb{P r}=0.843, C_{v}=5.375$, and the Van der Waals EOS is set as (8). 


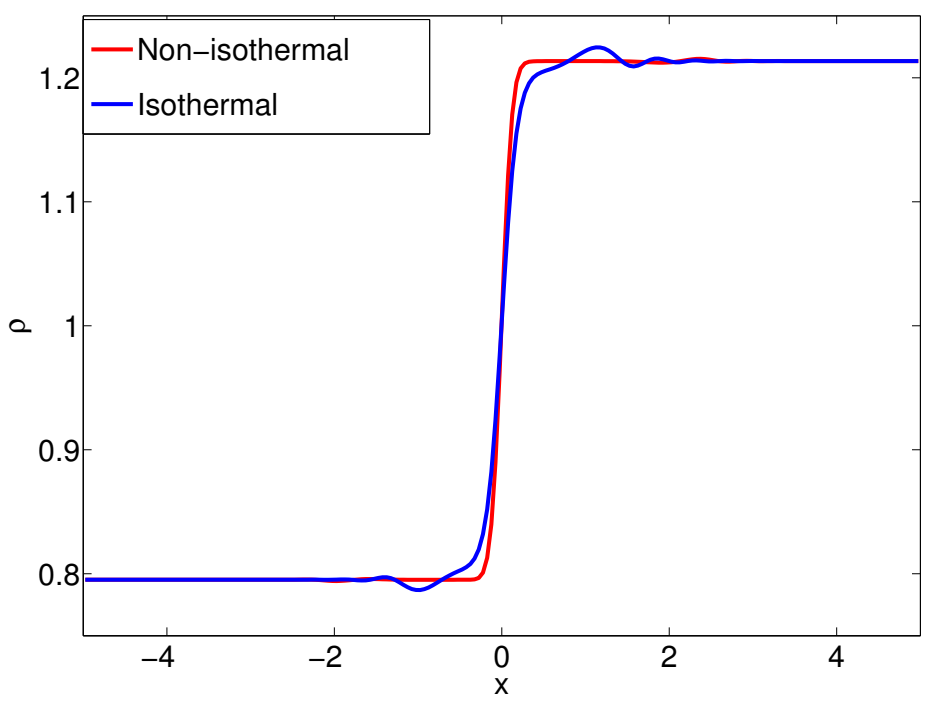

(a) density

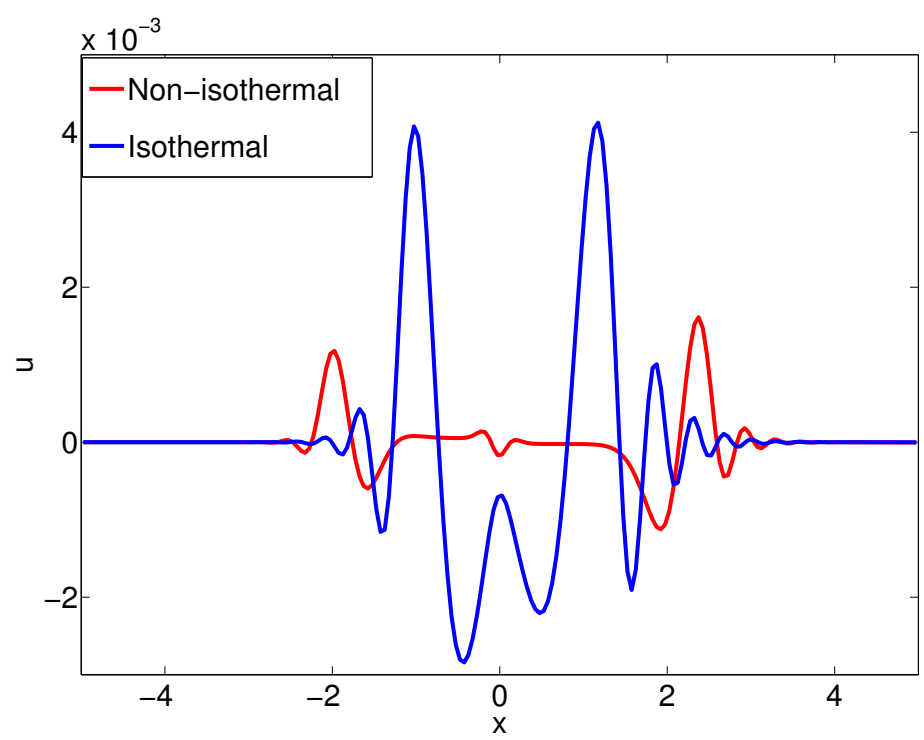

(b) velocity

Figure 17: One-dimensional static interface problem for the isothermal and non-isothermal NSK equations, numerical solutions obtained with the piecewise quadratic polynomials at $t=2.0$ on a mesh containing 400 elements. The physical parameters are chosen as $\mathbb{R} e=128.6, \mathbb{W} e=968.6, \mathbb{P r}=0.843, C_{v}=5.375$, and the Van der Waals EOS is set as $(8)$. 
where $\theta_{0}$ is a chosen constant, and $\rho_{1}, \rho_{2}$ are constants close to the critical densities for given $\theta_{0}$. These values will be specified in each test. The points $\mathbf{x}_{i}$ are equal to $\mathbf{x}_{1}=(0.4,0.5)$ and $\mathbf{x}_{2}=(0.78,0.5)$. The radii of the two bubbles are $r_{1}=0.25$ and $r_{2}=0.1$.

For the non-isothermal NSK equations with equation of state (8), the interface width (29) shows the following results:

- Given the initial temperature $\theta=0.989$, the critical densities are $\rho_{v}=$ $0.7952, \rho_{l}=1.2135$, and the interface width follows as

$$
d=\frac{2\left(\rho_{l}-\rho_{v}\right)}{\sqrt{\Delta f_{\max }}} \frac{1}{\sqrt{\mathbb{W} e}}=31.05 \frac{1}{\sqrt{\mathbb{W} e}} .
$$

Then the mesh size $h=\frac{2}{\sqrt{W e}}$ can be chosen.

- Given the initial temperature $\theta=0.95$, the critical densities are $\rho_{v}=$ 0.5790, $\rho_{l}=1.4617$, and the interface width follows as

$$
d=\frac{2\left(\rho_{l}-\rho_{v}\right)}{\sqrt{\Delta f_{\max }}} \frac{1}{\sqrt{\mathbb{W} e}}=14.42 \frac{1}{\sqrt{\mathbb{W} e}}
$$

Then the mesh size $h=\frac{1}{\sqrt{W e}}$ is a reasonable choice.

We use the LDG discretization for the non-isothermal NSK equations with bi-linear basis functions and the second order implicit Runge-Kutta time integration method (25) with stopping parameter $\eta_{k}=10^{-6}$ in (28). Similar to the isothermal case, the bubbles merge into one vapour bubble, which tends to be of circular shape later in time by capillarity and pressure forces. Choosing $\theta_{0}=0.989$, the initial condition is set as (39) with $\rho_{1}=0.795, \rho_{2}=$ 1.213. The LDG discretizations is used for the non-isothermal NSK equations with $\mathbb{W} e=34455$ on a mesh of $200^{2}$ square elements and a mesh of $100^{2}$ square elements. These two meshes lead to very similar results for mass conservation and entropy increase, see Figure 18. The process of coalescence computed for the non-isothermal NSK equations with initial condition (39) and $\rho_{1}=0.795, \rho_{2}=1.213, \theta_{0}=0.989$ on a mesh of $200^{2}$ elements is shown in Figures 19-21. Density and pressure along the line $y=0.5$ for two coalescing bubbles are computed with the LDG discretization of the non-isothermal NSK equations on a mesh with $100^{2}$ square elements, shown in Figure 22.

Given $\theta_{0}=0.95$, critical densities $\rho_{v}=0.5790, \rho_{l}=1.4617$ with a larger density ratio are found by (29) with equations of state (8). The initial condition is set as (39) with $\rho_{1}=0.579, \rho_{2}=1.462$ and $\theta_{0}=0.95$. The time 


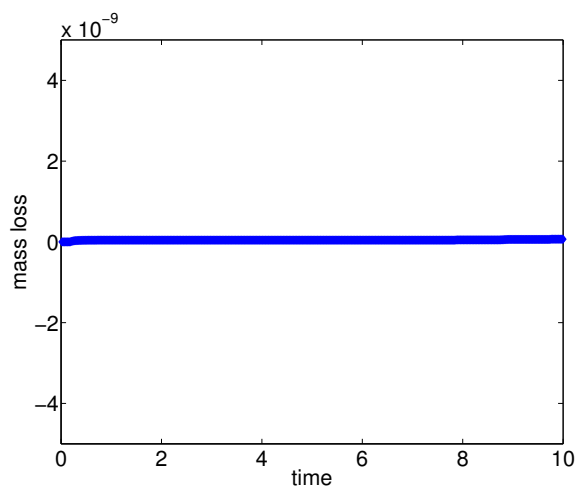

(a) mass loss

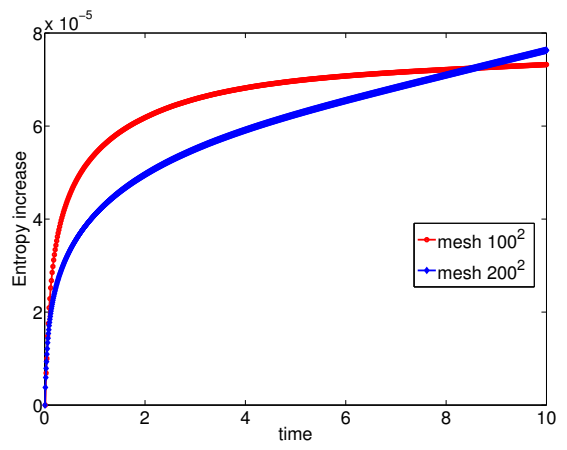

(b) entropy increase

Figure 18: Evolution of mass loss on a mesh of $100^{2}$ elements and entropy on meshes of $100^{2}$ and $200^{2}$ square elements as a function of time during the coalescence of two bubbles for the non-isothermal NSK equations with $\mathbb{R} e=950, \mathbb{W} e=34455, \mathbb{P r}=0.843, C_{v}=5.375$. The initial condition is set as (39) with $\rho_{1}=0.795, \rho_{2}=1.213$ and $\theta_{0}=0.989$.

evolution of the bubbles, mass and entropy are shown in Figures 23 and 25. The mass is conserved, and the entropy is a non-decreasing function of time apart from a small interval in which it is almost constant. For $\theta_{0}=0.92$, the critical densities are $\rho_{v}=0.479, \rho_{l}=1.587$. The initial condition is set as (39) with $\rho_{1}=0.479, \rho_{2}=1.587$. Figure 24 shows that stable results are obtained although the entropy does not increase during part of the calculation. A finer mesh is required to guarantee an increasing entropy in this case.

The behaviour of the numerical scheme for the Non-isothermal NSK equations is also studied when the initial densities are further away from the equilibrium densities $\rho_{l}, \rho_{v}$. Given $\theta_{0}=0.989, \rho_{1}=0.6, \rho_{2}=1.4$ is set in (39). Figures 26 shows the mass is conserved and entropy is increasing in time. Figure 27 shows the momentum in both directions and energy are conserved. Figure 28 presents the coalescence, similar results with Figure 19.

\section{Conclusions}

We developed local discontinuous Galerkin methods for the solution of the (non)-isothermal Navier-Stokes-Korteweg equations containing the Van der Waals equation of state and nonlinear third order density derivatives. The LDG methods are based on the conservative form of the NSK equations and are relatively simple compared to other available numerical discretizations 


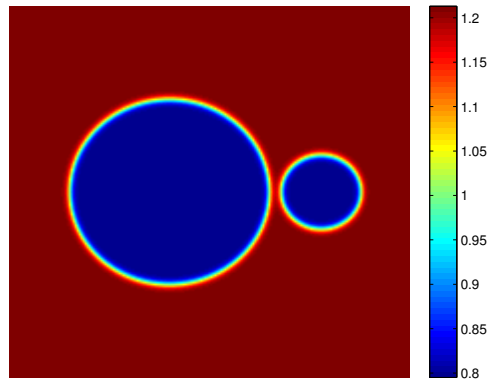

(a) time $t=0$.

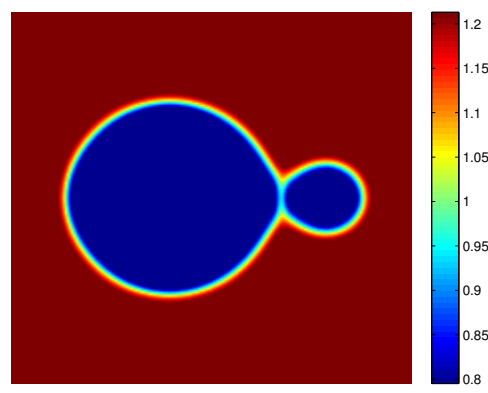

(c) time $t=6$.

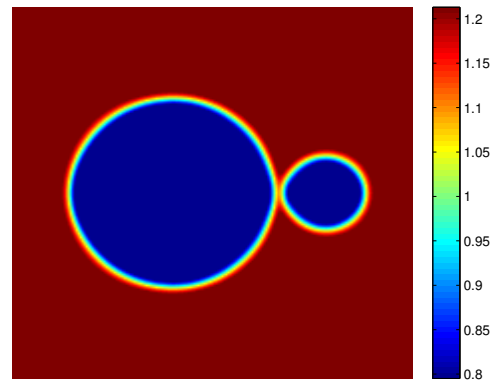

(b) time $t=3$.

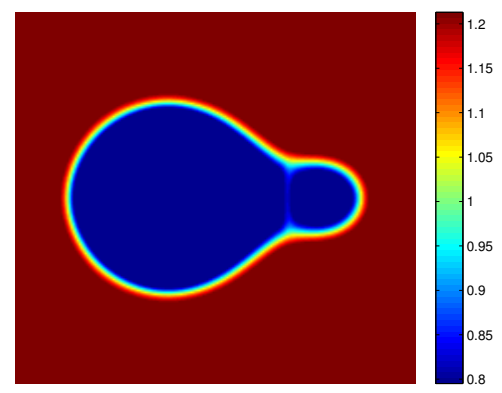

(d) time $t=9$.

Figure 19: Density $\rho$ for two coalescing bubbles computed with the LDG discretization of the non-isothermal NSK equations using piecewise linear polynomials on a mesh with $200^{2}$ square elements. The physical parameters are chosen as $\mathbb{R} e=950, \mathbb{W} e=34455, \mathbb{P r}=$ $0.843, C_{v}=5.375$, and the Van der Waals EOS is set as (8). The initial condition is set as (39) with $\rho_{1}=0.795, \rho_{2}=1.213$ and $\theta_{0}=0.989$. 


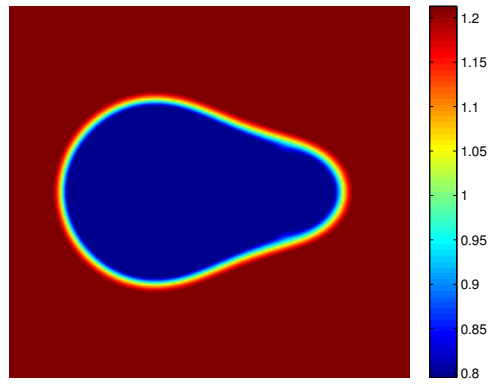

(a) time $t=12$.

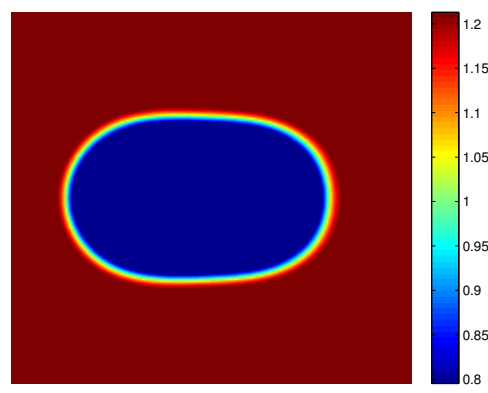

(c) time $t=18$.

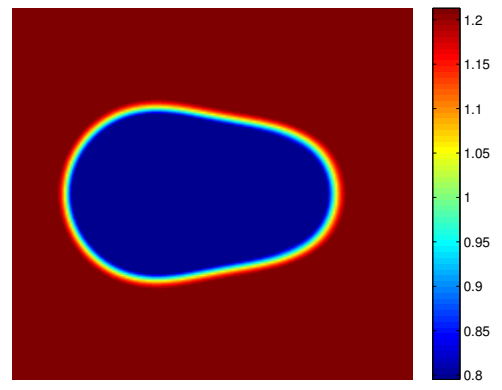

(b) time $t=15$.

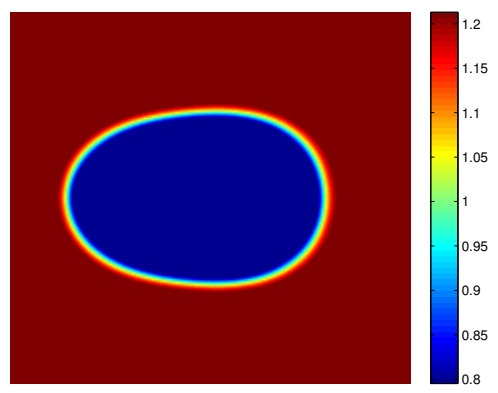

(d) time $t=21$.

Figure 20: Density $\rho$ for two coalescing bubbles computed with the LDG discretization of the non-isothermal NSK equations using piecewise linear polynomials with $200^{2}$ square elements. The physical parameters are chosen as $\mathbb{R} e=950, \mathbb{W} e=34455, \mathbb{P r}=0.843, C_{v}=$ 5.375 , and the Van der Waals EOS is set as (8). The initial condition is set as (39) with $\rho_{1}=0.795, \rho_{2}=1.213$ and $\theta_{0}=0.989$. 


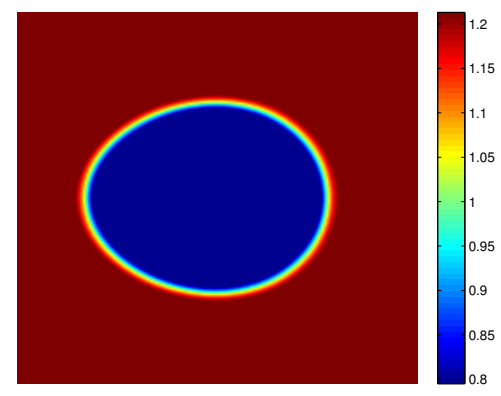

(a) time $t=28$.

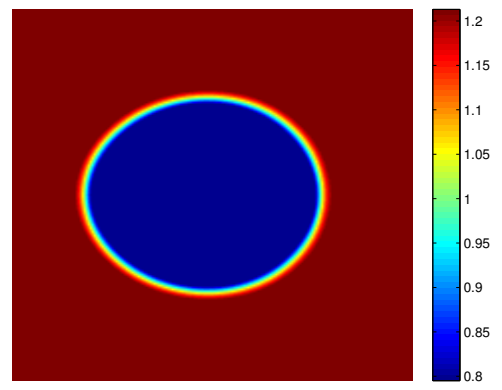

(b) time $t=30$.

Figure 21: Density $\rho$ for two coalescing bubbles computed with an LDG discretization of the non-isothermal NSK equations using piecewise linear polynomials functions with $200^{2}$ square elements. The physical parameters are chosen as $\mathbb{R} e=950, \mathbb{W} e=34455, \mathbb{P r}=$ $0.843, C_{v}=5.375$, and the Van der Waals EOS is set as (8). The initial condition is set as (39) with $\rho_{1}=0.795, \rho_{2}=1.213$ and $\theta_{0}=0.989$.

for the NSK equations. A diagonally implicit Runge-Kutta integration time method is used to integrate in time in order to deal with the severe time step restriction encountered for explicit time integration methods. The Jacobian matrix for the implicit Runge-Kutta method includes the extra variables for the higher order derivatives. The numerical experiments demonstrate the capabilities, accuracy and stability of the proposed LDG discretizations of the NSK equations. It is worthwhile to point out that the proposed LDG discretization is straightforward and works well for larger density ratios, but has limitations in the mesh required to obtain stable solutions and the correct energy or entropy behaviour.

In future research we will also consider the (non)-isothermal NSK equations for initial conditions that result in a larger elliptic region in the phase transition area. This will be combined with local mesh refinement to capture the interface more efficiently.

\section{Acknowledgements}

L.Tian acknowledges the China Scholarship Council (CSC) for giving the opportunity and financial support to study at the University of Twente in the Netherlands. Research of Yan Xu is supported by NSFC grant No.11371342, 


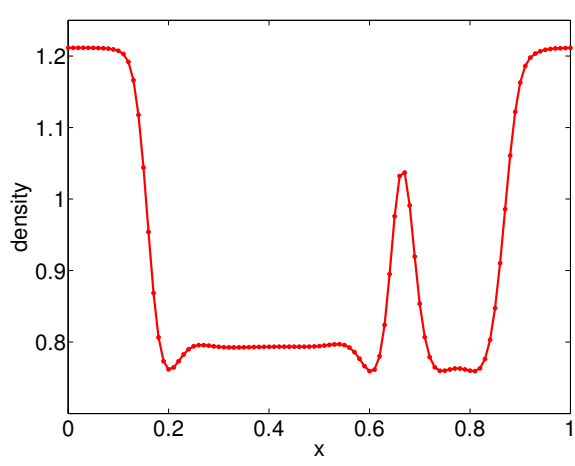

(a) time $t=5, \rho$ along $y=0.5$.

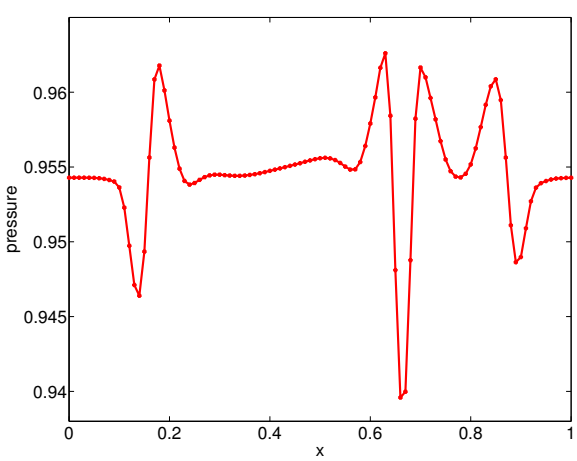

(c) time $t=5, p$ along $y=0.5$.

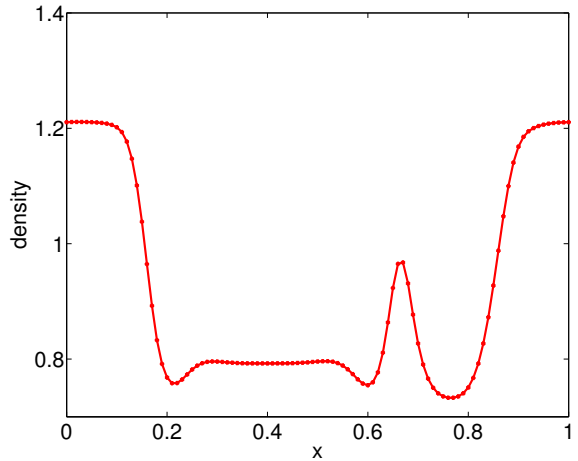

(b) time $t=10, \rho$ along $y=0.5$.

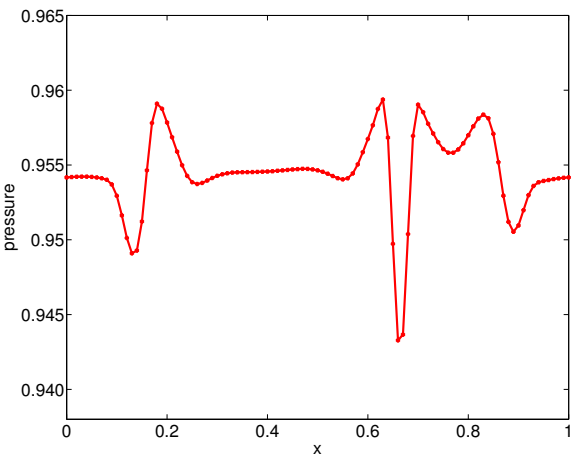

(d) time $t=10, p$ along $y=0.5$.

Figure 22: Density and pressure along $y=0.5$ for two coalescing bubbles computed with the LDG discretization of the non-isothermal NSK equations using piecewise linear polynomials on a mesh with $100^{2}$ square elements. The physical parameters are chosen as $\mathbb{R} e=950, \mathbb{W} e=34455, \mathbb{P r}=0.843, C_{v}=5.375$, and the Van der Waals EOS is set as (8). The initial condition is set as (39) with $\rho_{1}=0.795, \rho_{2}=1.213$ and $\theta_{0}=0.989$. 


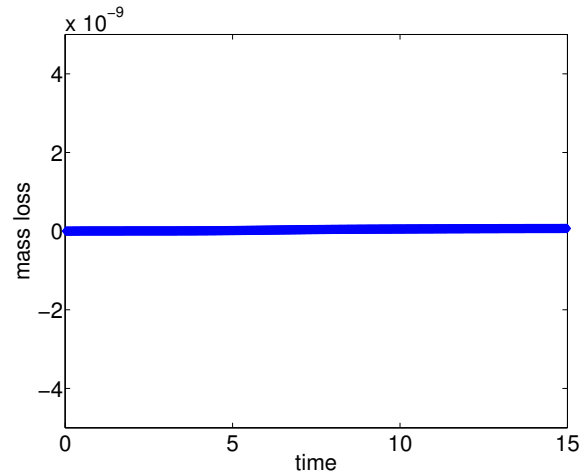

(a) mass loss

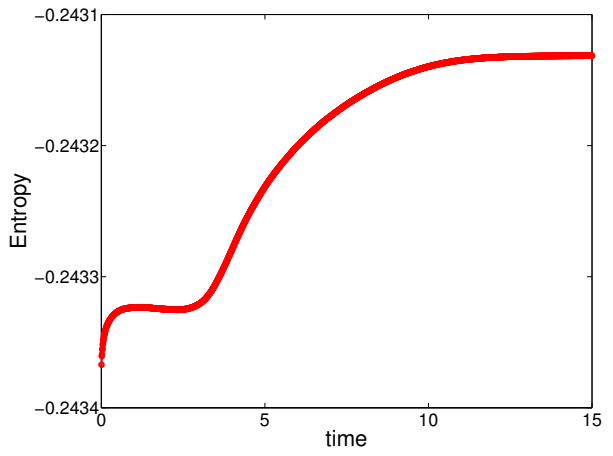

(b) entropy

Figure 23: Evolution of mass loss and entropy as a function of time during the coalescence of two bubbles for the non-isothermal NSK equations, the initial temperature set as $\theta=$ 0.95 , a mesh of $200^{2}$ square elements. The physical parameters are chosen as $\mathbb{R} e=$ 950, $\mathbb{W} e=34455, \mathbb{P r}=0.843, C_{v}=5.375$, and the Van der Waals EOS is set as (8). The initial condition is set as (39) with $\rho_{1}=0.579, \rho_{2}=1.462$ and $\theta_{0}=0.95$.

No.11031007, Fok Ying Tung Education Foundation No.131003. Research of J.J.W. van der Vegt was partially supported by the High-end Foreign Experts Recruitment Program (GDW201371001 68), while the author was in residence at the University of Science and Technology of China in Hefei, Anhui, China.

\section{Appendix}

In this Appendix we briefly discuss the derivation of the dimensionless form of the NSK equations and the definition of the dimensionless variables.

\section{Dimensionless form of the isothermal NSK equations}

The isothermal NSK equations are given by $[29,5,14]$

$$
\begin{array}{r}
\frac{\partial \rho}{\partial t}+\nabla \cdot(\rho \mathbf{u})=0, \\
\frac{\partial \rho \mathbf{u}}{\partial t}+\nabla \cdot(\rho \mathbf{u} \otimes \mathbf{u}+p \mathbf{I})-\nabla \cdot \tau-\nabla \cdot \xi=\mathbf{0},
\end{array}
$$




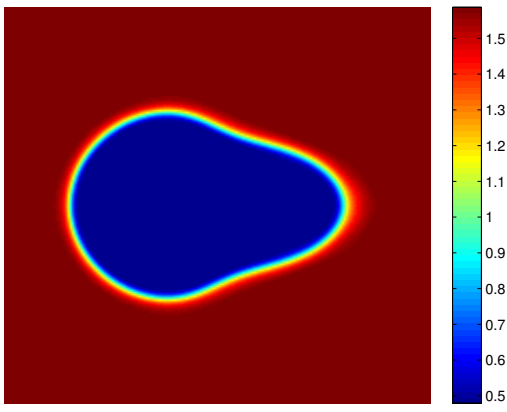

(a) $t=5$

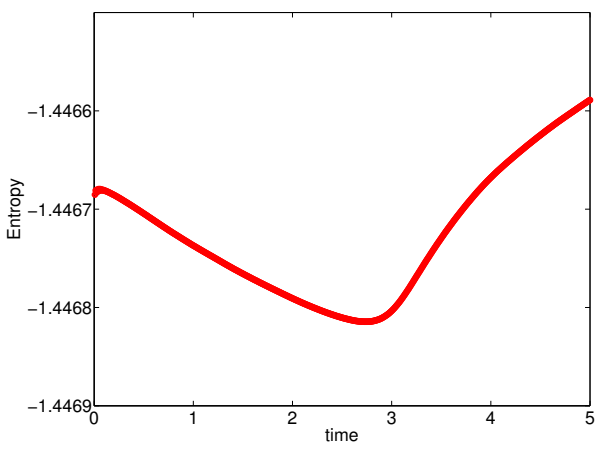

(b) Entropy

Figure 24: Density profile at $t=5.0$ and entropy as a function of time during the coalescence of two bubbles for the non-isothermal NSK equations, the initial temperature set as $\theta=0.92$, a mesh of $200^{2}$ square elements. The physical parameters are chosen as $\mathbb{R} e=950, \mathbb{W} e=34455, \mathbb{P r}=0.843, C_{v}=5.375$, and the Van der Waals EOS is set as (8). The initial condition is set as (39) with $\rho_{1}=0.479, \rho_{2}=1.587, \theta_{0}=0.92$.

where $\rho$ is the mass density, $\mathbf{u}$ the velocity. The viscous stress tensor $\tau$ and the Korteweg stress tensor are defined as

$$
\begin{array}{r}
\tau=\mu\left(\nabla \mathbf{u}+\nabla^{T} \mathbf{u}-\frac{2}{3} \nabla \cdot \mathbf{u I}\right), \\
\xi=\lambda\left(\left(\rho \triangle \rho+\frac{1}{2}|\nabla \rho|^{2}\right) \mathbf{I}-\nabla \rho \nabla^{T} \rho\right),
\end{array}
$$

with $\mu$ the viscosity coefficient and $\lambda$ the capillary coefficient. The thermodynamic pressure is defined as

$$
p=R b \frac{\rho \theta}{b-\rho}-a \rho^{2},
$$

with $\theta$ the temperature, $R$ the universal gas constant, $a, b$ positive constants depending on the fluid.

The equations are made dimensionless using the following reference variables for the mass density, temperature and pressure

$$
\rho_{c}=b, \theta_{c}=\frac{8 a b}{27 R}, p_{c}=a b^{2},
$$




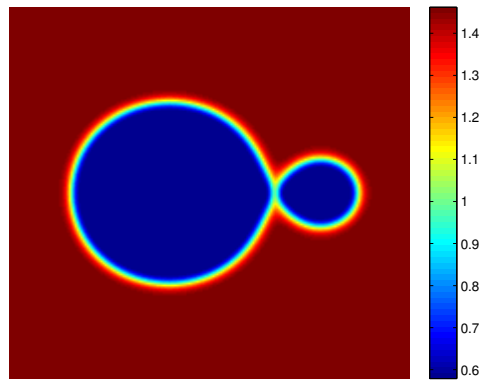

(a) time $t=3$.

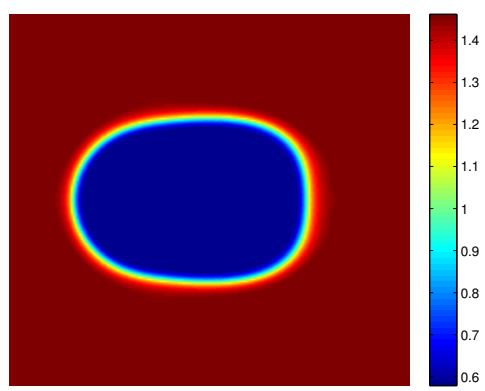

(c) time $t=9$.

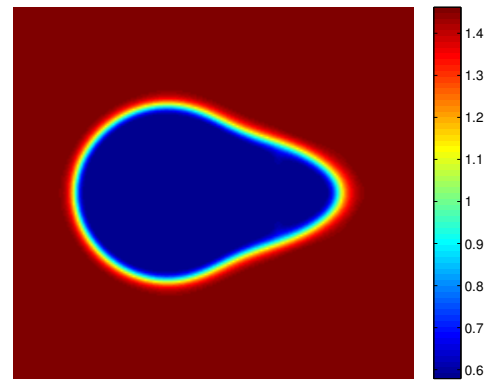

(b) time $t=6$.

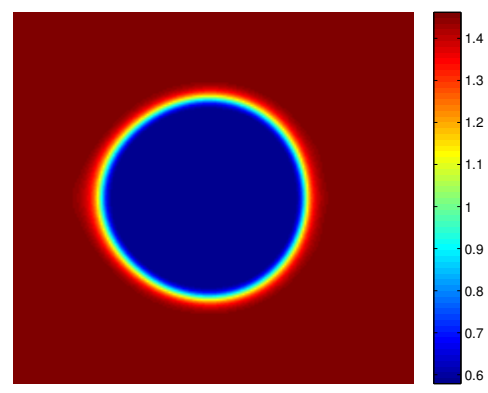

(d) time $t=15$.

Figure 25: Density $\rho$ for two coalescing bubbles computed with the LDG discretization of the non-isothermal NSK equations using piecewise linear polynomials on a mesh with $200^{2}$ square elements. The physical parameters are chosen as $\mathbb{R} e=950, \mathbb{W} e=34455, \mathbb{P r}=$ $0.843, C_{v}=5.375$, and the Van der Waals EOS is set as (8). The initial condition is set as (39) with $\rho_{1}=0.579, \rho_{2}=1.462$ and $\theta_{0}=0.95$ 


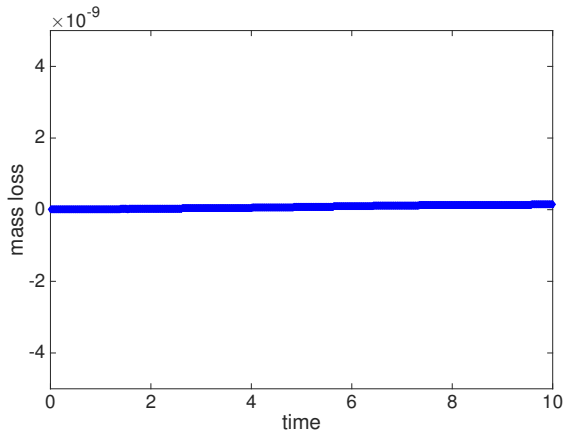

(a) mass loss

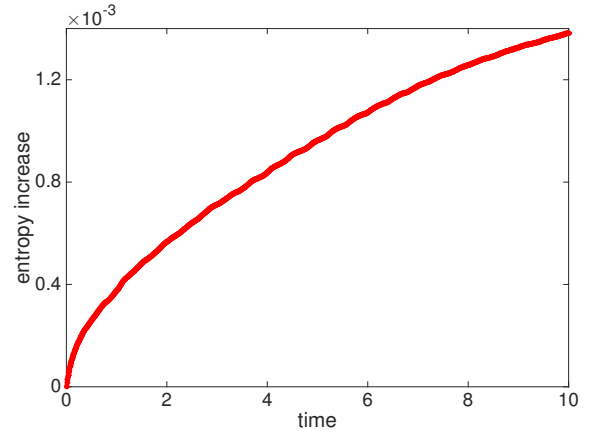

(b) entropy increase

Figure 26: Evolution of mass loss and entropy on a mesh of $200^{2}$ square elements as a function of time during the coalescence of two bubbles for the non-isothermal NSK equations, $\mathbb{R} e=950, \mathbb{W} e=34455, \mathbb{P r}=0.843, C_{v}=5.375$, and the Van der Waals EOS is set as (8). The initial condition is set as (39) with $\theta_{0}=0.989, \rho_{1}=0.6, \rho_{2}=1.4$.

The reference variable for the velocity is the average sound speed in the system $u_{c}=\sqrt{p_{c} / \rho_{c}}$ and the reference variable for time is $\frac{L}{u_{c}}$, with $L$ the reference length. The Reynolds and Weber numbers are then defined as

$$
\mathbb{R} e=\rho_{c} u_{c} L / \mu, \quad \mathbb{W} e=u_{c}^{2} L^{2} /\left(\rho_{c} \lambda\right) .
$$

Letting

$$
\rho=\rho_{c} \widetilde{\rho}, \mathbf{u}=u_{c} \widetilde{\mathbf{u}}, p=p_{c} \widetilde{p}, \theta=\theta_{c} \widetilde{\theta},
$$

the governing equations (40) and (42) then can be transformed into their dimensionless form, resulting in (1)-(3).

\section{Dimensionless form of the non-isothermal NSK equations}

The non-isothermal NSK equations are given by $[13,32]$

$$
\begin{array}{r}
\frac{\partial \rho}{\partial t}+\nabla \cdot(\rho \mathbf{u})=0, \\
\frac{\partial \rho \mathbf{u}}{\partial t}+\nabla \cdot(\rho \mathbf{u} \otimes \mathbf{u}+p \mathbf{I})-\nabla \cdot \tau-\nabla \cdot \xi=\mathbf{0}, \\
\frac{\partial(\rho E)}{\partial t}+\nabla \cdot((\rho E+p) \mathbf{u})-\nabla \cdot((\tau+\xi) \cdot \mathbf{u})+\nabla \cdot \mathbf{q}+\nabla \cdot \mathbf{j}_{E}=0
\end{array}
$$




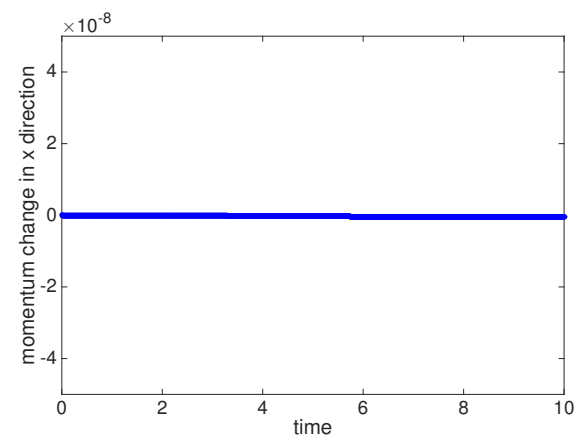

(a) momentum loss in $x$ direction

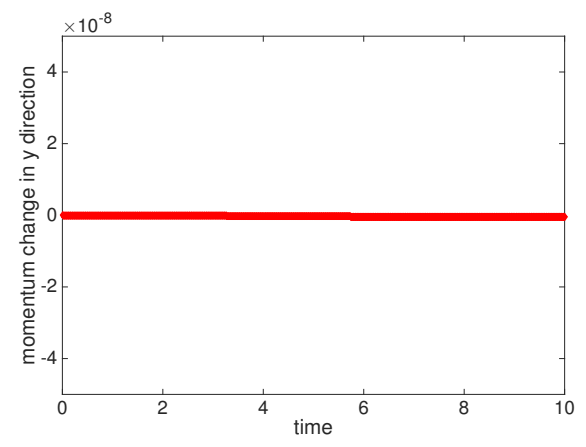

(b) momentum loss in $y$ direction

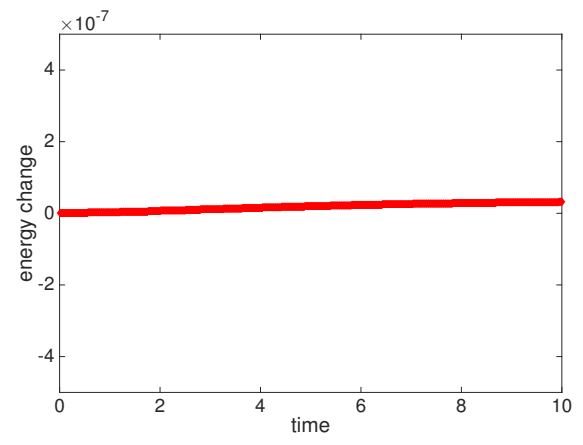

(c) energy loss

Figure 27: Evolution of momentum in $x$ and $y$ direction on a mesh of $200^{2}$ square elements as a function of time during the coalescence of two bubbles for the non-isothermal NSK equations, $\mathbb{R} e=950, \mathbb{W} e=34455, \mathbb{P r}=0.843, C_{v}=5.375$, and the Van der Waals EOS is set as (8). The initial condition is set as (39) with $\theta_{0}=0.989, \rho_{1}=0.6, \rho_{2}=1.4$. 


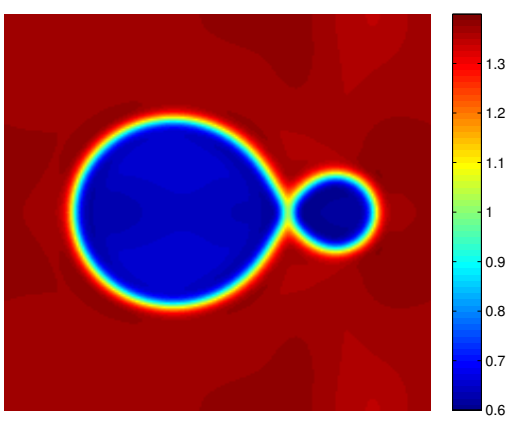

(a) time $t=4$.

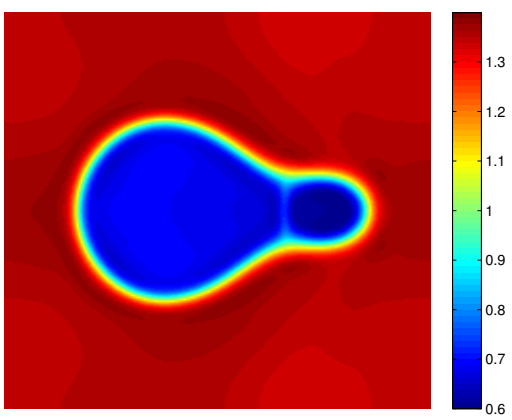

(c) time $t=8$.

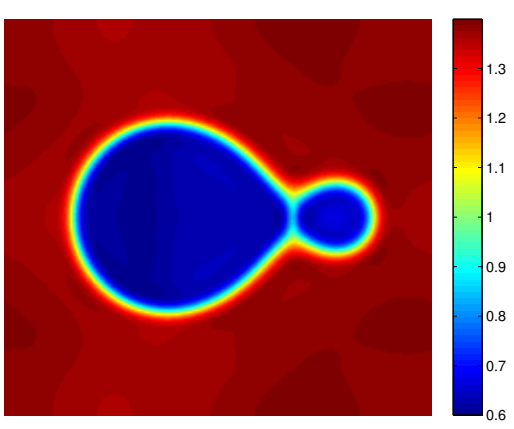

(b) time $t=6$.

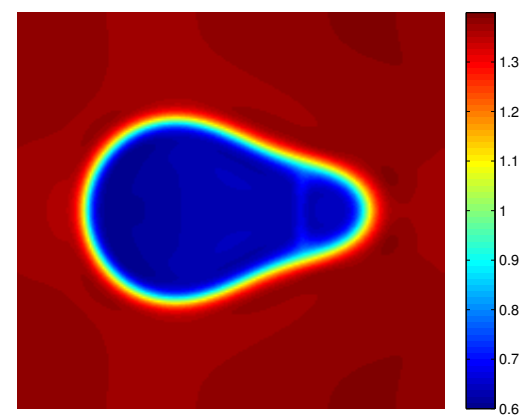

(d) time $t=10$.

Figure 28: Density $\rho$ for two coalescing bubbles computed with the LDG discretization of the non-isothermal NSK equations using piecewise linear polynomials on a mesh with $200^{2}$ square elements. The physical parameters are chosen as $\mathbb{R} e=950, \mathbb{W} e=34455, \mathbb{P r}=$ $0.843, C_{v}=5.375$, and the Van der Waals EOS is set as (8). The initial condition is set as (39) with $\theta_{0}=0.989, \rho_{1}=0.6, \rho_{2}=1.4$. 
with the definition of viscous stress tensor $\tau$ and the Korteweg stress tensor defined in (41). The total energy density is given by

$$
\rho E=\rho e+\frac{1}{2} \rho|\mathbf{u}|^{2}+\frac{1}{2} \lambda|\nabla \rho|^{2},
$$

with the specific internal energy $e$ defined as

$$
e=C_{v} \theta-\frac{a}{M^{2}} \rho
$$

The Van der Waals equation of state for the non-isothermal NSK equations (43) is given by

$$
p=\frac{R \theta \rho}{M-b \rho}-\frac{a}{M^{2}} \rho^{2},
$$

where $C_{v}$ is the specific heat at constant volume, $R$ the perfect gas constant, $M$ the molar mass of the fluid, $b$ the molar volume and $a$ a constant modelling the interactions between the fluid particles. The heat flux $\mathbf{q}$ and energy flux $\mathbf{j}_{E}$ through the interface in (43) are defined as

$$
\mathbf{q}=-K \nabla \theta, \quad \mathbf{j}_{E}=\lambda(\rho \nabla \cdot \mathbf{u}) \nabla \rho,
$$

with $K$ the thermal conductivity.

Note that the form of the equation of state (45) for the non-isothermal NSK equations is different from (42) for the isothermal NSK equations, though they have a similar shape.

The reference variables for the mass density, temperature and pressure are, respectively,

$$
\rho_{c}=M /(3 b), \theta_{c}=\frac{8 a}{27 R b}, p_{c}=a /\left(27 b^{2}\right) .
$$

The reference variable for the velocity is defined as $u_{c}=\sqrt{p_{c} / \rho_{c}}$ and the reference variable for time is $\frac{L}{u_{c}}$, with $L$ the reference length. The Reynolds and Weber numbers are then equal to

$$
\mathbb{R} e=\rho_{c} u_{c} L / \mu, \quad \mathbb{W} e=u_{c}^{2} L^{2} /\left(\rho_{c} \lambda\right) .
$$

The Prandtl number and the reduced heat capacity are defined as $\mathbb{P r}=$ $\mu C_{v} / K, \widetilde{C_{v}}=M C_{v} / R$.

The governing equations (43)-(46) then can be transformed into their dimensionless form, resulting in (6)-(9). 
[1] R. Abeyaratne and J.K. Knowles. Implications of viscosity and straingradient effects for the kinetics of propagating phase boundaries in solids. SIAM Journal on Applied Mathematics, 51:1205 - 1221, 1991.

[2] R. Alexander. Diagonally implicit Runge-Kutta methods for stiff ODE's. SIAM J. Numer. Anal., 14:1006 - 1021, 1977.

[3] D. M. Anderson, G. B. McFadden, and A. A. Wheeler. Diffuse-interface methods in fluid mechanics. Annu. Rev. Fluid Mech., 30:139 - 165, 1998.

[4] G.K. Batchelor. An Introduction to Fluid Dynamics. Cambridge University Press, 1967.

[5] M. Braack and A. Prohl. Stable discretization of a diffuse interface model for liquid-vapor flows with surface tension. ESAIM Math. Model. Numer. Anal., 47:401 - 420, 2013.

[6] D. Bresch, B. Desjardins, and C. K. Lin. On some compressible fluid models: Korteweg, lubrication, and shallow water systems. Comm. Partial Differential Equations, 28:843 - 868, 2003.

[7] J. W. Cahn and J. E. Hilliard. Free energy of a nonuniform system. I. Interfacial free energy. The Journal of chemical physics, 28:258 - 267, 1958.

[8] J. R. Cash. Diagonally implicit Runge-Kutta formulae with error estimates. IMA Journal of Applied Mathematics, 24:293 - 301, 1979.

[9] B. Cockburn and C. W. Shu. The local discontinuous Galerkin method for time-dependent convection-diffusion systems. SIAM J. Numer. Anal., 141:2440 - 2463, 1998.

[10] F. Coquel, D. Diehl, C. Merkle, and C. Rohde. Sharp and diffuse interface methods for phase transition problems in liquid-vapour flows. Numerical methods for hyperbolic and kinetic problems, 7:239 - 270, 2005.

[11] J. Crank. Free and Moving Boundary Problems. Oxford Univerrsity Press, 1997. 
[12] R. Danchin and B. Desjardins. Existence of solutions for compressible fluid models of Korteweg type. Ann. Inst. H. Poincar Anal. Non Linaire, 18:97 - 133, 2001.

[13] J. L. Desmarais and J. G. M. Kuerten. Open boundary conditions for the Diffuse Interface Model in 1-D. J. Comput. Phys., 263:393 - 418, 2014 .

[14] D. Diehl. Higher order schemes for simulation of compressible liquidvapor flows with phase change. PhD thesis, Universität Freiburg, 2007.

[15] S. C. Eisenstat and H. F. Walker. Globally convergent inexact Newton methods. SIAM Journal on Optimization, 4:393 - 422, 1994.

[16] J. Giesselmann, C. Makridakis, and T. Pryer. Energy consistent discontinuous Galerkin methods for the Navier-Stokes-Korteweg system. Mathematics of Computation, 83:2071-2099, 2014.

[17] H. Gomez, T. J. Hughes, X. Nogueira, and V. M. Calo. Isogeometric analysis of the isothermal Navier-Stokes-Korteweg equations. Comput. Methods Appl. Mech. Engrg., 199:1828 - 1840, 2010.

[18] J. Haink and C. Rohde. Local discontinuous Galerkin schemes for model problems in phase transition theory. Commun. Comput. Phys, 4:860893, 2008.

[19] E. Hairer and G. Wanner. Solving ordinary differential equation II. Stiff and differential-algebraic problems. Springer, 2002.

[20] B. Haspot. Existence of global weak solution for compressible fluid models of Korteweg type. Journal of Mathematical Fluid Mechanics, 13:223 - 249, 2011.

[21] H. Hattori and D. Li. Solutions for two-dimensional system for materials of Korteweg type. SIAM Journal on Mathematical Analysis, 25:85 - 98, 1994.

[22] H. Hattori and D. Li. The existence of global solutions to a fluid dynamic model for materials for Korteweg type. Journal of Partial Differential Equations, 9:323 - 342, 1996. 
[23] C. W. Hirt and B. D. Nichols. Volume of fluid (VOF) method for the dynamics of free boundaries. J. Comput. Phys., 39:201 - 225, 1981.

[24] D. Jamet, D. Torres, and J. U. Brackbill. On the theory and computation of surface tension: the elimination of parasitic currents through energy conservation in the second-gradient method. J. Comput. Phys., 182:262 $-276,2002$.

[25] C. T. Kelley. Iterative methods for linear and nonlinear equations. Society for Industrial and Applied Mathematics, 1995.

[26] M. Kotschote. Strong solutions for a compressible fluid model of Korteweg type. Ann. Inst. H. Poincar Anal. Non Linaire, 25:679 - 696, 2008 .

[27] M. Kotschote. Strong solutions to the compressible non-isothermal Navier-Stokes equations. Adv. Math. Sci. Appl., 22:319 - 347, 2012.

[28] M. Kotschote. Dynamical Stability of Non-Constant Equilibria for the Compressible Navier-Stokes Equations in Eulerian Coordinates. Commun. Math. Phys., 328:809 - 847, 2014.

[29] J. Liu, H. Gomez, J. A. Evans, T. J. Hughes, and C. M. Landis. Functional entropy variables: A new methodology for deriving thermodynamically consistent algorithms for complex fluids, with particular reference to the isothermal Navier-Stokes-Korteweg equations. J. Comput. Phys., 248:47 - 86, 2013.

[30] R. P. Pawlowski, J. N. Shadid, J. P. Simonis, and H. F. Walker. Globalization techniques for Newton-Krylov methods and applications to the fully coupled solution of the Navier-Stokes equations. SIAM review, 48:700 - 721, 2006.

[31] A. Pecenko, J. G. M. Kuerten, and C. W. M. van der Geld. A diffuseinterface approach to two-phase isothermal flow of a Van der Waals fluid near the critical point. Int. J. Multiphase Flow, 36:558 - 569, 2010.

[32] A. Pecenko, L. G. M. Van Deurzen, J. G. M. Kuerten, and C. W. M. van der Geld. Non-isothermal two-phase flow with a diffuse-interface model. Int. J. Multiphase Flow, 37:149 - 165, 2011. 
[33] C. Rohde. On local and non-local Navier-Stokes-Korteweg systems for liquid-vapour phase transitions. ZAMM Z. Angew. Math. Mech., 85:839 $-857,2005$.

[34] L. M. Skvortsov. Diagonally implicit Runge-Kutta methods for stiff problems. Comput. Math. Math. Phys., 46:2110 - 2123, 2006.

[35] L. Tian, Y. Xu, J. G. M. Kuerten, and J. J. W. Van der Vegt. A local discontinuous Galerkin method for the propagation of phase transition in solids and fluids. J. Sci. Comput., 59:688 - 720, 2014.

[36] G. Tryggvason, B. Bernard, E. Asghar, J. Damir, N. Al-Rawahi, W. Tauber, J. Han, S. Nas, and Y-J. Jan. A front-tracking method for the computations of multiphase flow. J. Comput. Phys., 169:708$759,2001$.

[37] Y. Xu and C. W. Shu. A local discontinuous Galerkin method for the Camassa-Holm equation. SIAM J. Numer. Anal., 46:1998 - 2021, 2008.

[38] Y. Xu and C.W. Shu. Local discontinuous Galerkin methods for highorder time-dependent partial differential equations. Commun. Comput. Phys., 7:1-46, 2010.

[39] J. Yan and C.W. Shu. A local discontinuous Galerkin method for KdV type equations. SIAM J. Numer. Anal., 40:769 - 791, 2002.

[40] P. Yue, J. J. Feng, C. Liu, and J. Shen. A diffuse-interface method for simulating two-phase flows of complex fluids. J. Fluid Mech., 515:293 $317,2004$. 